hep-th/0603243

MPP-2006-37

\title{
Matrix models for D-particle dynamics and the string/black hole transition
}

\author{
Johanna Erdmenger, Jeong-Hyuck Park and Corneliu Sochichiu \\ Max-Planck-Institut für Physik, Föhringer Ring 6, 80805 München, Germany \\ Electronic correspondence: jke, park, sochichi@mppmu.mpg.de
}

\begin{abstract}
For a generic two-dimensional 0A string background, we map the Dirac-Born-Infeld action to a matrix model. This is achieved using a canonical transformation. The action describes D0-branes in this background, while the matrix model has a potential which encodes all the information of the background geometry. We apply this formalism to specific backgrounds: For Rindler space, we obtain a matrix model with an upside-down quadratic potential, while for $A d S_{2}$ space, the potential is linear. Furthermore we analyze the black hole geometry with RR flux. In particular, we show that at the Hagedorn temperature, the resulting matrix model coincides with the one for the linear dilaton background. We interpret this result as a realization of the string/black hole transition.
\end{abstract}




\section{Introduction and summary}

As is well known, the end points of open strings are always attached to D-branes, and the understanding of D-brane dynamics can provide an alternative description of open strings. In particular, in noncritical two-dimensional 0A string theory, the spectrum of possible stable D-branes is restricted to D-particles. Therefore the particle dynamics of the D-branes should represent the two-dimensional open strings themselves. This essentially amounts to the holographic principle for two-dimensional string theories or the ' $A d S_{2} / \mathrm{CFT}_{1}$ ' correspondence $[1,2]$. Throughout this paper, we take this point of view as our guiding principle and provide evidence for its validity.

In the present paper, we derive matrix models from the one-dimensional Dirac-Born-Infeld (DBI) action with Chern-Simons (CS) term which describes D0-branes in two-dimensional 0A string backgrounds. The resulting matrix model is "non-relativistic" in the sense that the kinetic term is simply the velocity squared. All the information of the background geometry is encoded in the potential. Our map from the DBI-CS action to a matrix model corresponds to a canonical transformation in classical mechanics. No approximation such as the low velocity limit is taken. Essentially the canonical transformation is possible since the system is integrable, in the sense that there is only one dynamical variable matching the only conserved quantity, i.e. energy.

We apply our general formalism to a number of known two-dimensional backgrounds, including black hole backgrounds with flux. Within string theory, black holes are formed by condensation of D0-branes. We work in the regime where the pair interaction between D-particles is weak, and may therefore be replaced by the interaction with a self-consistent background, which in the present case is the black hole geometry. Therefore, we start with a probe analysis of the Born-Infeld action in the background, and obtain a corresponding "non-relativistic" action for a single particle. Then we generalize it to a gauged matrix model to describe a gas of D-particles. We show that in the gauged matrix model the pair interaction between particles is negligible, and hence the resulting matrix model is consistent with our probe analysis in the self-consistent background. Moreover, the gauging of the matrix model is consistent with the fact that D-particles are identical. Therefore we identify the gauged matrix model as an effective description of the black hole itself. This will be our second guiding principle. The above interpretation is in the spirit of understanding black holes in terms of falling matter outside the horizon, i.e. in the causally connected region. In this region the string coupling is bounded from above by the value at the horizon which is given by the inverse of the flux. Then, by taking a large value of flux, we can make quantum corrections negligible [3]. In this case, the classical Born-Infeld analysis for a probe brane can be trusted.

We also consider the linear dilaton background. Unlike the black hole backgrounds, in this case the string coupling is not bounded and the Born-Infeld analysis may not be trusted in the strong coupling region. In spite of this, the classical trajectory of the Born-Infeld action agrees with the conformal field theory result where the trajectory is obtained from the peak of the quantum wave 
function [4-6]. There is however an issue of localization, as discussed in [7].

We observe that the matrix model for the black hole background at the Hagedorn temperature is identical to the one for the linear dilaton background. This is a nontrivial result, as the two backgrounds are geometrically distinct and the expressions of the matrix variable in terms of the Liouville coordinate are different, as we will see in (4.8) and (5.12). We interpret our result as the 'string/black hole transition' at the Hagedorn temperature, which was initially proposed by Susskind for generic black holes [8-10], elaborated further in [11], and recently discussed for two-dimensional black holes in [7,12-15]. According to this proposal, there is a one-to-one correspondence between black hole and string states. Here we see an explicit realization of this physical picture: At the Hagedorn temperature, where the string coupling vanishes, the black hole matrix model coincides with the one for the linear dilaton background. The former is the matrix model for D-particles in the black hole background, which we assume to describe the black hole itself, while the latter describes fundamental strings. At the level of the D0 trajectory, the transition was already observed in a similar way in [13]. Here we describe the transition in terms of matrix models, taking particular care of the normalization of the potential.

The fact that the matrix models for two distinct backgrounds coincide in the vanishing string coupling regime suggests that this coincidence is a more generic phenomenon. This leads us to the conjecture that in the vanishing string coupling limit, the D0-brane dynamics or string theory itself becomes independent of background, such that the resulting matrix model is universally given by the matrix model with an upside-down quadratic potential, of which the coefficient is fixed in string units. In other words, in the vanishing string coupling limit the matrix model does not refer to any specific background geometry. In principle, this can be checked explicitly by calculating physical quantities, such as the spectrum or scattering amplitudes in different backgrounds.

Boundary states of D0-branes have been earlier studied in a coset conformal field theory at level $\kappa[7,13,16]$ (see also [3,6,17-24]). This background is generically different from ours, except at a single intersecting point $\kappa=1 / 2$, which in our case corresponds to the black hole at the Hagedorn temperature.

The organization and the summary of the present paper are as follows:

In Sec. 2] we review the exact black hole solutions in two-dimensional 0A string theory, and consider the near-horizon as well as two different weak coupling limits. We also set up our notations to express the solutions in terms of the black hole temperature.

In Sec. 3, we construct the canonical transformation which maps DBI-CS actions for Dparticles in a generic two-dimensional string background to matrix models. In the latter, the kinetic term is of canonical form as in non-relativistic mechanics, while the potential is nontrivial and contains all the information of the background geometry. The potential naturally decom- 
poses into DBI part and CS part. In particular, we establish the relation between the matrix variable and the Liouville coordinate for a generic background. We also analyze the isometry of the background, if present, and discuss its implication for the corresponding matrix model. We derive a necessary and sufficient condition for the existence of an isometry, and solve the Killing equations completely. We classify possible isometry groups and show that they are generically three-dimensional such as $\mathrm{SO}(1,2), \mathrm{SO}(3)$ or the Poincaré group. The isometry is inherited by the matrix model for the DBI sector and always gives rise to a $\mathrm{SO}(1,2)$ symmetry. An important result is that the isometry of the background geometry implies that the DBI potential is a polynomial, at most quadratic in the matrix variable. This is also consistent with the earlier work [25], where it was shown that only such matrix models allow for $\operatorname{OSp}(1 \mid 2, \mathbf{R})$ supersymmetric extension. We also show that the condition for the isometry is equivalent to the static equation of motion in Liouville field theory. This supports our proposal that our matrix models provide an appropriate description of noncritical two-dimensional 0A string theory.

Section 4 contains some simple applications. We apply the general formalism to Rindler space, $A d S_{2}$ and the linear dilaton backgrounds, and derive the corresponding matrix models. For Rindler space as well as the linear dilaton background, the resulting matrix models have an upside-down quadratic potential. For $A d S_{2}$ the potential is linear. The results for Rindler and $A d S_{2}$ spaces are interpreted as the first order approximation of the description of noncritical strings near the non-extremal and extremal black hole horizons.

In section [5] we carry out a detailed analysis of the matrix models for the exact black hole geometry. Firstly, we show that the matrix variable is positive semi-definite $X \geq 0$. Spatial infinity and outer horizon correspond to $X=0$ and $X=\infty$, respectively. For the black hole at the Hagedorn temperature, we find that the corresponding matrix model coincides with the one we obtained for the linear dilaton background. This corresponds to a realization of the string/black hole transition. Further, we conjecture that in the vanishing string coupling limit, irrespective of the background geometry, the matrix model is of universal form. We also consider the black hole near the Hagedorn temperature, and derive the corresponding matrix model with a nontrivial potential, involving an 'arctangent' function.

We continue by studying the near-horizon geometries of the extremal and non-extremal black holes by taking into account corrections to the matrix model potential up to the string length scale. The resulting matrix model depends on the black hole temperature explicitly. For the nonextremal black hole, it essentially agrees with the 'deformed matrix model' proposed by Jevicki and Yoneya [26]. On the other hand for the extremal black hole, the potential contains linear, square root and logarithmic terms.

Beyond the 0A gravity background, we also consider the black hole background in a coset conformal field theory at level $\kappa$. When $\kappa=1 / 2$, this geometry coincides with the one for the black 
hole at the Hagedorn temperature. We obtain the corresponding matrix model with $\kappa$-dependent potential. We identify the black hole temperature as function of $\kappa$, and find agreement with the conformal field theory result.

In the appendix we review how the $\mathrm{SO}(1,2)$ symmetry inherited from the isometry of the background geometry is realized in the matrix model, following [25]. The $\mathrm{SO}(1,2)$ exists if the potential consists of quadratic, linear and inverse square terms. Especially when the quadratic term is absent, the matrix model possesses $\mathrm{SO}(1,2)$ conformal symmetry. This applies to most of the matrix models we obtain, in particular to the Rindler, $A d S_{2}$, linear dilaton, non-extremal black hole and CFT background matrix models. However, for the extremal black hole case, there are logarithmic and square root contributions to the potential.

\section{Low energy effective action and black hole geometry}

The low energy effective action - in the Wilsonian sense - for the two-dimensional type 0A string theory reads

$$
S_{0 \mathrm{~A}}=S_{\mathrm{bulk}}+S_{\mathrm{brane}}+S_{\text {int. }} .
$$

Here $S_{\text {bulk }}$ is an action of two-dimensional gravity coupled to a closed string tachyon $T$ and a pair of RR field strengths $F^{(+)}$and $F^{(-)}$. Explicitly $[4,20,27,28]$,

$$
\begin{gathered}
S_{\text {bulk }}=-\int \mathrm{d}^{2} x \sqrt{-g}\left[\frac{1}{2 \kappa^{2}} e^{-2 \Phi}\left(\frac{8}{\alpha^{\prime}}+R+4(\nabla \Phi)^{2}-f_{1}(T)(\nabla T)^{2}+f_{2}(T)+\cdots\right)\right. \\
\left.-\frac{2 \pi \alpha^{\prime}}{4}\left(f_{3}(T)\left(F^{(+)}\right)^{2}+f_{3}(-T)\left(F^{(-)}\right)^{2}\right)+\cdots\right] .
\end{gathered}
$$

As there are two different RR gauge potentials, there are also two distinct D0-branes, D0 ${ }^{(+)}$and $\mathrm{D}^{(-)}[4]$. Accordingly the action for the D-branes, $S_{\text {brane }}$, decomposes into $S_{\text {brane }}^{(+)}+S_{\text {brane }}^{(-)}$, each of which corresponds to a non-Abelian Dirac-Born-Infeld-like action for relativistic particles,

$$
S_{\text {brane }}^{( \pm)}=-\frac{1}{\kappa \sqrt{\alpha^{\prime}}} \int \mathrm{d} s \text { "Tr" }\left(e^{-\Phi} \sqrt{-g_{\mu \nu} \dot{x}^{( \pm) \mu} \dot{x}^{( \pm) \nu}}\right) .
$$

Here we write "Tr" with a double quotation mark, as the precise form of the $2 D 0 \mathrm{~A}$ non-Abelian Dirac-Born-Infeld action is not known. However, in the weak coupling region which we consider in the subsequent, only the Abelian part contributes.

Finally, $S_{\text {int. }}$ describes the interactions between the bulk modes and the brane modes, and includes Chern-Simons-like terms for the coupling of the RR gauge potential to the D-particles,

$$
S_{\text {int. }}=\sqrt{2} \int \mathrm{d} s \operatorname{Tr}\left(C_{\mu}^{(+)} \dot{x}^{(+) \mu}\right)+\operatorname{Tr}\left(C_{\mu}^{(-)} \dot{x}^{(-) \mu}\right)+\cdots .
$$


In particular, in the presence of $N^{(+)}$number of $\mathrm{D} 0^{(+)}$static branes, the equation of motion for $C_{t}^{(+)}$reads with $x^{0} \equiv t, x^{1} \equiv \phi$,

$$
\pi \alpha^{\prime} \partial_{\phi}\left(f_{3}(T) F^{(+)}\right)=\sqrt{2} \sum_{n}^{N^{(+)}} \delta\left(\phi-\phi_{n}(t)\right) .
$$

Integrating this over $\phi$, we see that the flux is quantized. One may also ask about contributions of the brane action (2.3) to other equations of motion for the bulk NS fields. However, since we focus on the weak string coupling region we can neglect these.

In $[29,30]$ (see also $[20,22,23]$ ), black hole solutions are obtained for the equations of motion derived from the bulk action (2.2) for vanishing tachyon. Requiring the tachyon to vanish $(T \equiv 0)$ implies that the fluxes must be equal, ${ }^{1}[24,28]$,

$$
\left(F^{(+)}\right)^{2}=\left(F^{(-)}\right)^{2}=\frac{Q^{2}}{2 \pi^{2} \alpha^{\prime 2}}, \quad Q=N^{(+)}=N^{(-)} .
$$

The flux then plays the role of a negative cosmological constant, and the solutions of the equations of motion of the bulk action (2.2) are given by, after setting $2 \kappa^{2} \equiv 1$,

$$
\begin{array}{ll}
\mathrm{d} s^{2}=-l(\phi) \mathrm{d} t^{2}+\frac{1}{l(\phi)} \mathrm{d} \phi^{2}, & l(\phi)=1+e^{2 \sqrt{2} \phi m_{s}}\left(\frac{\left(g_{s} Q\right)^{2}}{4 \sqrt{2} \pi} \phi m_{s}-\xi\right), \\
\Phi(\phi)=\sqrt{2} \phi m_{s}+\Phi_{0}, & C_{t}^{( \pm)}(\phi)=-\frac{Q}{\sqrt{2} \pi \alpha^{\prime}} \phi+C_{t}^{( \pm)}(0), \quad C_{\phi}^{( \pm)}=0 .
\end{array}
$$

Here $m_{s}=\alpha^{\prime-\frac{1}{2}}$ is the string mass scale, $\xi$ is a dimensionless free parameter, and $\Phi_{0}$ is the vev of the dilaton which gives the string coupling at $\phi=0$,

$$
g_{s}=e^{\Phi_{0}} .
$$

The horizon, $\phi_{h}$, is, by definition, located at $l\left(\phi_{h}\right)=0$, so that we can express the free parameter $\xi$ by the horizon,

$$
\xi=e^{-2 \sqrt{2} \phi_{h} m_{s}}+\frac{\left(g_{s} Q\right)^{2}}{4 \sqrt{2} \pi} \phi_{h} m_{s} .
$$

As a function of $\phi_{h}, \xi$ is bounded below, ${ }^{2}$

$$
\xi \geq\left.\xi\right|_{\text {extremal }}=\frac{\left(g_{s} Q\right)^{2}}{16 \pi}\left[1-\ln \left(\frac{\left(g_{s} Q\right)^{2}}{16 \pi}\right)\right],
$$

\footnotetext{
${ }^{1}$ The normalization of $S_{\text {int. }}$ has been merely chosen to match the result of [4] where the flux is precisely the number of D-particles.

${ }^{2}$ The case $\xi<\left.\xi\right|_{\text {extremal }}$ corresponds to a naked singularity such that classical gravity breaks down. We exclude this possibility.
} 
and generically there are two horizons, the inner horizon $\phi_{h}^{\text {in }}$, and the outer horizon $\phi_{h}^{\text {out }}$, satisfying $\phi_{h}^{\text {out }} \leq \phi_{h}^{\text {in }}$, as the region $\phi \rightarrow-\infty$ corresponds to the asymptotically flat spatial infinity. When the bound on $\xi$ (2.10) is saturated, the horizon assumes the critical value

$$
\left.\phi_{h}\right|_{\text {extremal }}=-\sqrt{\frac{\alpha^{\prime}}{8}} \ln \left(\frac{\left(g_{s} Q\right)^{2}}{16 \pi}\right) .
$$

This corresponds to the extremal black hole, where the two horizons coincide. In general, we have

$$
\phi_{h}^{\text {out }} \leq\left.\phi_{h}\right|_{\text {extremal }} \leq \phi_{h}^{\text {in }} .
$$

Further, it is convenient to introduce the following parameters: the distance to the horizon and the derivative of $l(\phi)$ at the horizon,

$$
u \equiv \phi-\phi_{h}, \quad \varepsilon:=\left.\sqrt{\alpha^{\prime}} \partial_{\phi} l(\phi)\right|_{\phi=\phi_{h}}=2 \sqrt{2}\left(\frac{\left(g_{s} Q\right)^{2}}{16 \pi} e^{2 \sqrt{2} \phi_{h} m_{s}}-1\right) .
$$

Note that the two horizons, the inner and the outer, can be distinguished by the sign of $\varepsilon$, i.e. $\varepsilon^{\text {in }}>0$ for the inner horizon and $\varepsilon^{\text {out }}<0$ for the outer horizon. The temperature of the black hole is then given by $\left|\varepsilon^{\text {out }}\right|$ at the outer horizon,

$$
T_{\mathrm{BH}}:=-\left.\frac{1}{4 \pi} \partial_{\phi} l(\phi)\right|_{\phi=\phi_{h}^{\text {out }}}=\frac{m_{s}}{4 \pi}\left|\varepsilon^{\text {out }}\right|=\frac{m_{s}}{\sqrt{2} \pi}\left(1-\frac{\left(g_{s} Q\right)^{2}}{16 \pi} e^{2 \sqrt{2} \phi_{h}^{\text {out }} m_{s}}\right),
$$

which is bounded by the Hagedorn temperature from above,

$$
T_{\mathrm{BH}} \leq T_{\text {Hag. }}=\frac{m_{s}}{\sqrt{2} \pi} .
$$

Note that in all the expressions above, the dependence on the flux and the string coupling is through their product $g_{s} Q$ only.

Instead of $\Phi_{0}$ and $\xi$, we may take $\Phi\left(\phi_{h}\right)$ and $\varepsilon$ as alternative free parameters. Then with $\Phi(\phi)=\sqrt{2} u m_{s}+\Phi\left(\phi_{h}\right)$, we can rewrite the black hole solution (2.7) as

$$
\begin{aligned}
l(\phi) & =1+\left[(2 \sqrt{2}+\varepsilon) u m_{s}-1\right] e^{2 \sqrt{2} u m_{s}} \\
& =\varepsilon_{u m}+(4+2 \sqrt{2} \varepsilon)\left(u m_{s}\right)^{2}+\left(\frac{16 \sqrt{2}}{3}+4 \varepsilon\right)\left(u m_{s}\right)^{3}+\mathcal{O}\left[\left(u m_{s}\right)^{4}\right] .
\end{aligned}
$$

Note that the above formula holds for both the inner and outer horizons, i.e. the same function $l(\phi)$ allows for two different expressions. However, henceforth we will focus on the region outside 
the outer horizon so that $\varepsilon^{\text {out }} \leq 0$ and $u \leq 0$. This ensures that, from (2.12), the string coupling outside the outer horizon is bounded from above by the value for the extremal black hole [3]

$$
e^{\Phi\left(\phi_{h}^{\text {out }}\right)} \leq e^{\Phi\left(\left.\phi_{h}\right|_{\text {extremal }}\right)}=\frac{4 \sqrt{\pi}}{Q} .
$$

Thus, for large $Q$ the string coupling is small outside the outer horizon.

We now consider various weak coupling limits as well as the near-horizon limits of the exact black hole solution (2.7).

1. Rindler space:

For the non-extremal black hole solutions $T_{\mathrm{BH}} \neq 0$, the near outer horizon geometry $u<0$ is two-dimensional Rindler space from (2.16), [22]

$$
\mathrm{d} s^{2}=4 \pi T_{\mathrm{BH}} u \mathrm{~d} t^{2}-\frac{1}{4 \pi T_{\mathrm{BH}} u} \mathrm{~d} u^{2}
$$

2. $\mathbf{A d S}_{2}$ :

For the extremal black hole, $T_{\mathrm{BH}}=0$, the near-horizon geometry of the extremal black hole is $A d S_{2}$,

$$
\mathrm{d} s^{2}=-4\left(u m_{s}\right)^{2} \mathrm{~d} t^{2}+\frac{1}{4\left(u m_{s}\right)^{2}} \mathrm{~d} u^{2} .
$$

\section{Black hole at the Hagedorn temperature:}

Taking $g_{s} \rightarrow 0$ in (2.7) with the choice $\xi \neq 0$, the solution (2.7) reduces to the metric for the black hole at the Hagedorn temperature [31],

$$
\mathrm{d} s^{2}=-\left(1-e^{2 \sqrt{2} u m_{s}}\right) \mathrm{d} t^{2}+\frac{1}{1-e^{2 \sqrt{2} u m_{s}}} \mathrm{~d} u^{2}, \quad \Phi-\Phi\left(\phi_{h}\right)=\sqrt{2} u m_{s} .
$$

\section{Linear dilaton background:}

Taking $g_{s} \rightarrow 0$ in (2.7), this time choosing $\xi=0$ (or alternatively in the asymptotically flat region $\phi \rightarrow-\infty)$, the solution (2.7) becomes the linear dilaton background,

$$
\mathrm{d} s^{2}=-\mathrm{d} t^{2}+\mathrm{d} \phi^{2}, \quad \Phi=\sqrt{2} \phi m_{s}+\Phi_{0} .
$$

Note that the string coupling given by $e^{\Phi}=g_{s} e^{\sqrt{2} \phi m_{s}}$ grows as $\phi$ becomes large. One should bear in mind that in the strong coupling region the gravity action (2.2) is subject to quantum corrections and the solution (2.7) may not be valid. This restricts the validity of the linear dilation background (2.21) to $\phi \ll \sqrt{\alpha^{\prime}}\left|\ln g_{s}\right|$. However, in contrast to the linear dilaton background, for the generic black hole solution we consider, the string coupling is bounded from above (2.17) 
outside of the outer horizon. The strong coupling region is inaccessible for a fiducial observer.

In addition to the above black hole solutions of the gravity action (2.2), we also consider 'black hole' background in a coset conformal field theory at level $\kappa$, studied in $[7,13,16]$

$$
\mathrm{d} s^{2}=\kappa \alpha^{\prime}\left(-\tanh ^{2} \rho \mathrm{d} \tau^{2}+\mathrm{d} \rho^{2}\right), \quad \Phi=-\ln \cosh \rho+\Phi_{h}
$$

When $\kappa=1 / 2$, this coincides with the black hole background at the Hagedorn temperature (2.20), while for generic $\kappa \neq 1 / 2$, it is not a classical solution of the gravity action (2.2). The dimensionless coordinates $\tau, \rho$ are related to our time and Liouville coordinates $t, u$ through, with $\alpha^{\prime}=m_{s}^{-2}$,

$$
t m_{s}=\sqrt{\kappa} \tau, \quad \sqrt{2} u m_{s}=-\ln \cosh \rho,
$$

such that the horizon lies at $\rho=0(u=0)$, and at the spatial infinity $\rho=+\infty(u=-\infty)$ we have the normalization $g_{t t}=-1$. In terms of $t, u,(2.22)$ can be rewritten as

$$
\mathrm{d} s^{2}=-\left(1-e^{2 \sqrt{2} u m_{s}}\right) \mathrm{d} t^{2}+\frac{2 \kappa}{1-e^{2 \sqrt{2} u m_{s}}} \mathrm{~d} u^{2}, \quad \Phi=\sqrt{2} u m_{s}+\Phi\left(\phi_{h}\right) .
$$

Hence when $\kappa=1 / 2$, this reduces to (2.20). Further, in [7] it was shown that the wave function in the conformal field theory does not spread over a region but has a sharp localization when $\kappa=$ $1 / 2{ }^{3}$ This also justifies our classical analysis to take D-particle as a point particle. Applying our matrix model analysis to this additional background will allow us to compare with the conformal field theory result.

\section{Matrix model for the two-dimensional DBI-CS action}

\subsection{Non-relativistic formulation of the DBI-CS action}

We consider $N$ D-particles on generic two-dimensional backgrounds, in view of considering the black hole solution (2.7). We take the string coupling $g_{s}$ to be small such that the backreaction is negligible, and the interaction among the $N$ D-particles is switched off. For weak string coupling it is sufficient to consider a single D-particle treated as a probe. Its dynamics is governed by the Abelian Dirac-Born-Infeld action (2.3) with Chern-Simons term (2.4).

\footnotetext{
${ }^{3} \kappa$ is related to " $Q$ " in $[7]$ by " $Q$ " $=\sqrt{2 / \kappa}$.
} 
In a gauge where the metric is diagonal $g_{t \phi} \equiv 0$, and the world line parameter is identified with the target space time, $s \equiv t$, the action for D0-brane dynamics is of the general form

$$
S_{D 0}=\int \mathrm{d} t\left[\mp m_{s} \sqrt{\mathcal{E}_{t}(t, \phi)^{2} \mp \mathcal{E}_{\phi}(t, \phi)^{2} \dot{\phi}^{2}}+C_{t}(t, \phi)+C_{\phi}(t, \phi) \dot{\phi}\right]
$$

where we take the 'zweibein' for the effective metric $G_{\mu \nu} \equiv e^{-2 \Phi} g_{\mu \nu}$ to be

$$
\mathcal{E}_{t}^{2}:=e^{-2 \Phi}\left|g_{t t}\right|>0, \quad \mathcal{E}_{\phi}^{2}:=e^{-2 \Phi}\left|g_{\phi \phi}\right|>0 \text {. }
$$

The upper sign is for the Minkowskian spacetime, while the lower one is for the Euclidean space. Henceforth we focus on the static backgrounds, i.e. no explicit time dependence in the backgrounds, $\mathcal{E}_{t}(\phi), \mathcal{E}_{\phi}(\phi), C_{t}(\phi), C_{\phi}(\phi)$ as in the black hole solution (2.7). Consequently the last term in the action drops out, and the Hamiltonian reads

$$
H_{D 0}=H_{\mathrm{DBI}}-C_{t}(\phi)
$$

where $H_{\mathrm{DBI}}$ is the Hamiltonian for the DBI action. With the canonical momentum for $\phi$,

$$
p_{\phi}=\frac{m_{s} \dot{\phi} \mathcal{E}_{\phi}^{2}}{\sqrt{\mathcal{E}_{t}^{2} \mp \mathcal{E}_{\phi}^{2} \dot{\phi}^{2}}},
$$

it reads

$$
H_{\mathrm{DBI}}= \pm m_{s}\left(\left(\frac{1}{\mathcal{E}_{t}}\right)^{2} \mp\left(\frac{\dot{\phi} \mathcal{E}_{\phi}}{\mathcal{E}_{t}^{2}}\right)^{2}\right)^{-\frac{1}{2}}= \pm m_{s} \sqrt{\mathcal{E}_{t}^{2} \pm\left(\frac{p_{\phi} \mathcal{E}_{t}}{m_{s} \mathcal{E}_{\phi}}\right)^{2}}
$$

We define a new variable $X$ by

$$
X(\phi):=\frac{1}{g_{\hat{s}}} \int_{\phi_{0}}^{\phi} \mathrm{d} \phi^{\prime} \frac{\mathcal{E}_{\phi}\left(\phi^{\prime}\right)}{\mathcal{E}_{t}\left(\phi^{\prime}\right)^{2}}
$$

satisfying $\dot{X}=\dot{\phi} \mathcal{E}_{\phi} / \mathcal{E}_{t}^{2}$. This will turn out to be the matrix model variable, and Eq.(3.6) establishes the relation between the matrix variable and the Liouville coordinate for generic backgrounds, as we see below. Here we introduce an dimensionless parameter $g_{\hat{s}}$ which is a priori an arbitrary constant. However, the natural choice is to identify it with the string coupling, such that $X$ in (3.6) is independent of the string coupling. Hence, near the horizon region of black holes we set

$$
g_{\hat{s}} \equiv e^{\Phi\left(\phi_{h}^{\text {out }}\right)} .
$$


Since $\mathcal{E}_{t}, \mathcal{E}_{\phi}$ are non-negative definite, $X(\phi)$ is monotonically increasing with $\phi$. In terms of the new coordinates, $(t, X)$, the effective metric is

$$
e^{-2 \Phi} \mathrm{d} s^{2}=e^{-2 \Phi} g_{\mu \nu} \mathrm{d} x^{\mu} \mathrm{d} x^{\nu}=\mp \mathcal{E}_{t}^{2} \mathrm{~d} t^{2}+g_{\hat{s}}^{2} \mathcal{E}_{t}^{4} \mathrm{~d} X^{2}
$$

Now we rewrite (3.3) as

$$
0=\dot{X}^{2} \mp \frac{1}{g_{\hat{s}}^{2} \mathcal{E}_{t}(\phi(X))^{2}} \pm \frac{m_{s}^{2}}{g_{\hat{s}}^{2}\left[H_{D 0}+C_{t}(\phi(X))\right]^{2}} .
$$

From (3.9), it follows that the D0 dynamics on the generic static backgrounds has an alternative dual description as a non-relativistic particle motion,

$$
\mathcal{L}_{\text {nonrel. }}=\frac{1}{2} \dot{X}^{2} \pm \frac{1}{2 g_{\hat{s}}^{2} \mathcal{E}_{t}(\phi(X))^{2}} \mp \frac{m_{s}^{2}}{2 g_{\hat{s}}^{2}\left[H_{D 0}+C_{t}(\phi(X))\right]^{2}} .
$$

Here 'non-relativistic' merely refers to the fact that the kinetic term in (3.10) is non-relativistic. The dynamics given by (3.10) is restricted to the surface of vanishing energy in the phase space. The equations of motion for $X$ in both systems, the DBI action (3.1) and the non-relativistic action (3.10), are equivalent, as one can see easily by taking the time derivative of (3.9). ${ }^{4}$ This corresponds to a canonical transformation in classical mechanics of the form

$$
\left(\phi, p_{\phi}\right) \Longrightarrow\left(X(\phi), P_{X}=\frac{p_{\phi}}{\mathcal{E}_{t} \sqrt{m_{s}^{2} \mathcal{E}_{\phi}^{2} \pm p_{\phi}^{2}}}\right)
$$

where the new Hamiltonian after the transformation is given by half of the right hand side of (3.9) with $\dot{X} \equiv P_{X}$.

Further we consider the dynamics of the D0-brane gas. The above single particle nonrelativistic Lagrangian has a natural generalization to the Yang-Mills mechanics, i.e. to the matrix

\footnotetext{
${ }^{4}$ However, it is worthwhile to note that the above equivalence between the relativistic and non-relativistic systems is a special property of one dimension. In one dimension these are integrable systems where the number of dynamical variables is one, the same as the number of conserved quantities. In higher dimensions, one might consider

$$
" \mathcal{L} "=\left(\sum_{j=1}^{D} \frac{1}{2} \dot{X}^{j 2}\right) \pm \frac{1}{2 g_{\hat{s}}^{2} \mathcal{E}_{t}^{2}} \mp \frac{m_{s}^{2}}{2 g_{\hat{s}}^{2}\left(H_{D 0}+C_{t}\right)^{2}}, \quad X^{j}:=\frac{1}{g_{\hat{s}}} \int_{\phi_{0}^{j}}^{\phi^{j}} \mathrm{~d} \phi^{j \prime} \frac{\mathcal{E}_{\phi^{j}}\left(\phi^{\prime}\right)}{\mathcal{E}_{t}\left(\phi^{\prime}\right)^{2}},
$$

but in general this Lagrangian does not lead to equations of motion for relativistic particles. This would require integrability.
} 
model with $\mathrm{U}(N)$ gauge symmetry for the description of $N$ D-particles,

$$
\begin{aligned}
& \mathcal{L}_{\text {M.M. }}=\mathcal{L}_{\text {M.M. for DBI }}+\mathcal{L}_{\text {M.M. for CS }} \\
& \mathcal{L}_{\text {M.M. for DBI }}=m_{s} \operatorname{tr}\left[\frac{1}{2}\left(D_{t} X\right)^{2} \pm \frac{1}{2 g_{\hat{s}}^{2} \mathcal{E}_{t}(\phi(X))^{2}}\right] \\
& \mathcal{L}_{\text {M.M. for CS }}=m_{s} \operatorname{tr}\left[\mp \frac{1}{2\left[\mathcal{H}+g_{\hat{s}} m_{s}^{-1} C_{t}(\phi(X))\right]^{2}}\right] .
\end{aligned}
$$

Here $X$ is a Hermitian matrix of which the eigenvalues represent the positions of the D-particles. Eq.(3.12) is the generic form of the matrix model we discuss. In the subsequent we compute the potential for different background geometries.

The matrix model (3.12) describes the D-particle dynamics. Moreover, it is also the holographic dual of two-dimensional 0A string theory. As before, the dynamics is restricted to zero energy. The fluctuations around zero energy correspond to string excitations in the given background.

In (3.12) the ordinary time derivative is replaced by the covariant time derivative, with a non-dynamical gauge field $A$,

$$
D_{t} X=\dot{X}-i[A, X]
$$

in order to allow for the gauge symmetry,

$$
X \longrightarrow U^{-1} X U, \quad A \longrightarrow U^{-1} A U+i U^{-1} \partial_{t} U, \quad U \in \mathrm{U}(N) .
$$

The equation of motion for the auxiliary gauge field $A$ is a secondary first-class constraint. This implies that the physical states are in the gauge singlet sector.

There are two reasons justifying the gauging introduced in (3.13). The first is related to the path integral formulation of the above gauged matrix model. After choosing the diagonal gauge for $X$, the Vandermonde determinant appears as a Faddeev-Popov determinant. Then integrating out the gauge field $A$ precisely cancels the Vandermonde determinant [32]. The cancellation implies the absence of any pair interaction between D-particles. Hence, the D-particle is only subject to the force originating from the background geometry as given by the potential in (3.12). This is consistent with our probe analysis approach where the pair interaction is negligible.

The second reason for the gauging is related to the indistinguishability of the D-particles. The diagonalization of the $X$ is not unique; the Weyl group of $\mathrm{U}(N)$ in (3.14) acts by permuting the eigenvalues of $X$. Physically, this is just the fact that $D$-particles are identical particles, as 
noted by Witten [33]. In matrix models, gauging the $\mathrm{U}(N)$ symmetry naturally takes care of the ambiguity in the diagonalization. Different diagonalizations correspond to the same gauge orbit and hence to the same physical state (see [34] for further discussion). The gauging of the matrix model is also consistent with the gauge theory description of $D$-brane dynamics (see e.g. [21]).

In (3.12) we also introduce a dimensionless parameter, $\mathcal{H}$, related to the average of the $\mathrm{D}$ particle energy,

$$
\mathcal{H} \equiv \frac{g_{\hat{s}}}{m_{s}}\left\langle H_{D 0}\right\rangle \text {. }
$$

When the D0-branes are in thermal equilibrium with the background geometry, we can interpret $\mathcal{H}$ as the ensemble average. Moreover, $\mathcal{H}$ should be tuned as a function of the temperature, such that the thermodynamical energy of the matrix model vanishes. This is necessary for consistency with the fact that the dynamics is constrained to the surface of vanishing energy.

If there is no contribution from the Chern-Simons term, as in the $Q=0$ case, or for D-particles not charged under the given RR flux, e.g. $\mathrm{D} 0^{(+)}$on $C^{(-)}$flux, the matrix model $\mathcal{L}_{\text {M.M. in (3.12) }}$ reduces to the DBI matrix model,

$$
\mathcal{L}_{\mathrm{M} . \mathrm{M} . \text { for DBI }}=m_{s} \operatorname{tr}\left[\frac{1}{2}\left(D_{t} X\right)^{2} \pm \frac{1}{2 g_{\hat{s}}^{2} \mathcal{E}_{t}(\phi(X))^{2}}\right]
$$

The CS contribution given by the last equation of (3.12) becomes trivial, i.e. depends on $\mathcal{H}$ only, and the dynamics is now constrained to the Fermi surface,

$$
E_{\mathrm{FS}} \equiv \mp \frac{m_{s} N}{2 \mathcal{H}^{2}}=\mp \frac{m_{s}^{3} N}{2 g_{\hat{s}}^{2}\left\langle H_{D 0}\right\rangle^{2}}
$$

Again the upper and lower signs refer to the Minkowskian and the Euclidean signatures. In our conventions, $X$ has the dimension of length, while $\mathcal{L}_{\text {M.M. }}$ and $H_{\text {M.M. }}$ have mass dimension, and hence the action is dimensionless. Since $\left\langle H_{D 0}\right\rangle^{2}>0$, the Fermi surface energy given by (3.17) is negative for the Minkowskian signature. ${ }^{5}$

\footnotetext{
${ }^{5}$ In our normalization, $E_{\mathrm{FS}} \propto m_{s} N$. However, the overall factor of our matrix model is a priori arbitrary due to the restriction to zero energy. In analogy to standard Yang-Mills theory, we may rescale $X$ such that the resulting Lagrangian acquires an extra factor $g_{s}^{-1}$. Then the Fermi sea level is proportional to $g_{s}^{-1}$ as in [35].
} 


\subsection{Isometry of the background geometry, matrix model and Liouville theory}

The explicit form of the DBI potential, $\mp \frac{1}{2}\left[g_{\hat{s}} \mathcal{E}_{t}(\phi(X))\right]^{-2}$ in (3.12) as a function of $X$ can in principle be obtained after solving (3.6) for the inverse of $X(\phi)$. Alternatively, using the chain rule one can obtain the power series expansion of the potential in $X$, as done later in (3.35). However, remarkably, as we show in this subsection, if the background geometry with the effective metric $e^{-2 \Phi} g_{\mu \nu}$ admits a nontrivial isometry, the DBI potential must be a polynomial at most quadratic in $X$. Moreover, matrix models with at most quadratic potential, even with arbitrary time dependent coefficients, possess an $\mathrm{SO}(1,2)$ symmetry as Noether symmetry [25].

We focus on the static backgrounds with the effective metric,

$$
G_{\mu \nu}=e^{-2 \Phi} g_{\mu \nu}=\left(\begin{array}{cc}
\mp \mathcal{E}_{t}(\phi)^{2} & 0 \\
0 & \mathcal{E}_{\phi}(\phi)^{2}
\end{array}\right)_{\mu \nu},
$$

and analyze its isometry, if any,

$$
\mathcal{L}_{V} G_{\mu \nu}=0
$$

where $V$ is a Killing vector. As we restrict on the static backgrounds, there exits at least one isometry given by the time translational symmetry. Later, we discuss the implication of the isometry for the corresponding matrix model.

For the static background, (3.2), the Killing equations are explicitly

$$
\partial_{\phi} V^{\phi} \mathcal{E}_{\phi}+V^{\phi} \partial_{\phi} \mathcal{E}_{\phi}=0, \quad V^{\phi} \partial_{\phi} \mathcal{E}_{t}+\partial_{t} V^{t} \mathcal{E}_{t}=0, \quad \partial_{\phi} V^{t} \mathcal{E}_{t}^{2} \mp \partial_{t} V^{\phi} \mathcal{E}_{\phi}^{2}=0
$$

It turns out that one can solve the Killing equations analytically. The first relation implies $V^{\phi}(t, \phi)=\mathcal{E}_{\phi}^{-1}(\phi) f(t)$, which further gives along other two relations,

$$
\ddot{f}(t)=\Lambda(\phi) f(t), \quad \Lambda(\phi):=\mp \frac{\mathcal{E}_{t}^{2}}{\mathcal{E}_{\phi}} \partial_{\phi}\left(\frac{\partial_{\phi} \mathcal{E}_{t}}{\mathcal{E}_{t} \mathcal{E}_{\phi}}\right) .
$$

Thus, for a nontrivial solution to exist, the consistency requires

$$
\frac{\mathrm{d} \Lambda(\phi)}{\mathrm{d} \phi}=0 \quad \text { or } \quad \Lambda=\text { constant } .
$$

This is the necessary and sufficient condition for the zweibein, $\mathcal{E}_{t}(\phi), \mathcal{E}_{\phi}(\phi)$, to admit any nontrivial isometry, apart from the time translation. Provided this condition satisfied, one can obtain the most general solutions for the Killing vector. It turns out that they are given with three free parameters which we denote by $c_{+}, c_{-}, c_{0}$. This implies that the isometry group is three-dimensional. 
First, when $\Lambda \neq 0$ the Killing vector is of the general form,

$$
V^{t}=\frac{\partial_{\phi} \mathcal{E}_{t}}{\mathcal{E}_{t} \mathcal{E}_{\phi}}\left(c_{+} f_{+}(t)+c_{-} f_{-}(t)\right)+c_{0}, \quad V^{\phi}=-\frac{1}{\mathcal{E}_{\phi}}\left(c_{+} \dot{f}_{+}(t)+c_{-} \dot{f}_{-}(t)\right),
$$

where $f_{+}(t), f_{-}(t)$ are the two distinct solutions of the second order differential equation,

$$
\ddot{f}_{ \pm}(t)=f_{ \pm}(t) \Lambda
$$

Explicitly, for $\Lambda>0$,

$$
f_{+}(t)=\cosh (\sqrt{\Lambda} t), \quad f_{-}(t)=\sinh (\sqrt{\Lambda} t)
$$

while for $\Lambda<0$,

$$
f_{+}(t)=\cos (\sqrt{|\Lambda|} t), \quad f_{-}(t)=\sin (\sqrt{|\Lambda|} t) .
$$

In particular, the parameter $c_{0}$ amounts to the time translational symmetry of the static background, corresponding to the DBI Hamiltonian, $H_{\mathrm{DBI}}$. In order to identify the isometry algebra we take the generators, from (3.23) for $\Lambda \neq 0$, as

$$
\mathcal{J}_{+}:=-i\left(\frac{\partial_{\phi} \mathcal{E}_{t}}{\mathcal{E}_{t} \mathcal{E}_{\phi}} f_{+} \partial_{t}-\frac{1}{\mathcal{E}_{\phi}} \dot{f}_{+} \partial_{\phi}\right), \quad \mathcal{J}_{-}:=-i\left(\frac{\partial_{\phi} \mathcal{E}_{t}}{\mathcal{E}_{t} \mathcal{E}_{\phi}} f_{-} \partial_{t}-\frac{1}{\mathcal{E}_{\phi}} \dot{f}_{-} \partial_{\phi}\right), \quad H_{\mathrm{DBI}}:=-i \partial_{t}
$$

and obtain the commutator relations

$$
\left[\mathcal{J}_{+}, \mathcal{J}_{-}\right]=-i \Omega \sqrt{|\Lambda|} H_{\mathrm{DBI}}, \quad\left[H_{\mathrm{DBI}}, \mathcal{J}_{+}\right]=-i \operatorname{sign}(\Lambda) \sqrt{|\Lambda|} \mathcal{J}_{-}, \quad\left[H_{\mathrm{DBI}}, \mathcal{J}_{-}\right]=-i \sqrt{|\Lambda|} \mathcal{J}_{+}
$$

Here $\operatorname{sign}(\Lambda)$ denotes the sign of $\Lambda$, and $\Omega$ is a constant given by

$$
\Omega:=\left(\frac{\partial_{\phi} \mathcal{E}_{t}}{\mathcal{E}_{t} \mathcal{E}_{\phi}}\right)^{2}+\frac{1}{\mathcal{E}_{\phi}} \partial_{\phi}\left(\frac{\partial_{\phi} \mathcal{E}_{t}}{\mathcal{E}_{t} \mathcal{E}_{\phi}}\right), \quad \partial_{\phi} \Omega=0
$$

The constant property follows from the consistency condition of the isometry (3.21).

Now we turn to the case $\Lambda=0$, where the Killing vector is of the general form

$$
V^{t}=\frac{\partial_{\phi} \mathcal{E}_{t}}{\mathcal{E}_{t} \mathcal{E}_{\phi}}\left(\frac{1}{2} c_{+} t^{2}+c_{-} t\right) \mp\left(\int \mathrm{d} \phi \frac{\mathcal{E}_{\phi}}{\mathcal{E}_{t}^{2}}\right) c_{+}+c_{0}, \quad V^{\phi}=-\frac{1}{\mathcal{E}_{\phi}}\left(c_{+} t+c_{-}\right) .
$$

The corresponding generators are

$$
\mathcal{J}_{+}^{\prime}:=-i\left[\left(\frac{\partial_{\phi} \mathcal{E}_{t}}{2 \mathcal{E}_{t} \mathcal{E}_{\phi}} t^{2} \mp \int \mathrm{d} \phi \frac{\mathcal{E}_{\phi}}{\mathcal{E}_{t}^{2}}\right) \partial_{t}-\frac{t}{\mathcal{E}_{\phi}} \partial_{\phi}\right], \quad \mathcal{J}_{-}^{\prime}:=-i\left(\frac{\partial_{\phi} \mathcal{E}_{t}}{\mathcal{E}_{t} \mathcal{E}_{\phi}} t \partial_{t}-\frac{1}{\mathcal{E}_{\phi}} \partial_{\phi}\right)
$$


and $H_{\mathrm{DBI}}=-i \partial_{t}$, as before. In the case of $\Lambda=0$, the following two quantities are constants,

$$
\frac{\partial_{\phi} \mathcal{E}_{t}}{\mathcal{E}_{t} \mathcal{E}_{\phi}}:=\Omega^{\prime}, \quad \frac{1}{\mathcal{E}_{t}^{2}}+2 \frac{\partial_{\phi} \mathcal{E}_{t}}{\mathcal{E}_{t} \mathcal{E}_{\phi}} \int \mathrm{d} \phi \frac{\mathcal{E}_{\phi}}{\mathcal{E}_{t}^{2}} .
$$

Further, if the former is not zero, one can set the latter to vanish by choosing the integration constant properly. Accordingly, for $\Omega^{\prime} \neq 0$ we have the commutator relations

$$
\left[\mathcal{J}_{+}^{\prime}, \mathcal{J}_{-}^{\prime}\right]=i \Omega^{\prime} \mathcal{J}_{+}^{\prime}, \quad\left[H_{\mathrm{DBI}}, \mathcal{J}_{+}^{\prime}\right]=-i \mathcal{J}_{-}^{\prime}, \quad\left[H_{\mathrm{DBI}}, \mathcal{J}_{-}^{\prime}\right]=-i \Omega^{\prime} \mathcal{J}_{+}^{\prime},
$$

and for $\Omega^{\prime}=0$,

$$
\left[\mathcal{J}_{+}^{\prime}, \mathcal{J}_{-}^{\prime}\right]= \pm i \mathcal{E}_{t}^{-2} H_{\mathrm{DBI}}, \quad\left[H_{\mathrm{DBI}}, \mathcal{J}_{+}^{\prime}\right]=-i \mathcal{J}_{-}^{\prime}, \quad\left[H_{\mathrm{DBI}}, \mathcal{J}_{-}^{\prime}\right]=0 .
$$

Both of them can be identified as Poincaré algebra.

All together, we have the classification of the possible isometry group for two-dimensional

\begin{tabular}{|c|c|c|c|}
\hline \multicolumn{2}{|c|}{$\Lambda, \Omega, \Omega^{\prime}$} & & Isometry group \\
\hline$\Lambda>0$ & $\Omega>0$ & : & $\mathrm{SO}(1,2)$ \\
\hline$\Lambda>0$ & $\Omega=0$ & : & Minkowskian Poincaré group \\
\hline$\Lambda>0$ & $\Omega<0$ & : & $\mathrm{SO}(1,2)$ \\
\hline$\Lambda<0$ & $\Omega>0$ & : & $\mathrm{SO}(1,2)$ \\
\hline$\Lambda<0$ & $\Omega=0$ & : & Euclidean Poincaré group \\
\hline$\Lambda<0$ & $\Omega<0$ & : & $\mathrm{SO}(3)$ \\
\hline$\Lambda=0$ & $\Omega^{\prime}>0$ & : & Minkowskian Poincaré group \\
\hline$\Lambda=0$, & $\Omega^{\prime}=0$ & $\cdot$ & Poincaré group with the same signature as the target space \\
\hline$\Lambda=0$, & $\Omega^{\prime}<0$ & : & Euclidean Poincaré group \\
\hline
\end{tabular}
static backgrounds, as summarized in Table 1. In particular, $H_{\mathrm{DBI}}$ corresponds to a so $(1,1)$ generator if $\Lambda>0$, and so(2) generator if $\Lambda<0$.

Table 1: Classification of possible isometry group

In the remaining of this subsection, we discuss the implication of the isometry to the corresponding matrix model. From (3.6), (3.21), using the chain rule, we get

$$
\frac{\partial}{\partial X}\left( \pm \frac{1}{2 g_{\hat{s}}^{2} \mathcal{E}_{t}(\phi(X))^{2}}\right)=\mp \frac{\partial_{\phi} \mathcal{E}_{t}}{g_{\hat{s}} \mathcal{E}_{t} \mathcal{E}_{\phi}}:=\rho(\phi), \quad \frac{\partial^{2}}{\partial X^{2}}\left( \pm \frac{1}{2 g_{\hat{s}}^{2} \mathcal{E}_{t}(\phi(X))^{2}}\right)=\Lambda
$$

Remarkably this shows that when the background geometry with the effective metric, $e^{-2 \Phi} g_{\mu \nu}$ (3.18), admits an isometry, apart from the time translational symmetry, the potential in the DBI 
matrix model must be a polynomial at most quadratic in $X$,

$$
\mathcal{L}_{\text {M.M. for DBI }}=\operatorname{tr}\left[\frac{1}{2}\left(D_{t} X\right)^{2} \pm\left(2 g_{\hat{s}}^{2} \mathcal{E}_{t}^{2}\right)^{-1}\right]=\operatorname{tr}\left[\frac{1}{2}\left(D_{t} X\right)^{2}+\frac{1}{2} \Lambda X^{2}+\rho\left(\phi_{0}\right) X+\text { constant }\right] .
$$

Moreover, since $\mathcal{E}_{t}^{2}$ is positive definite, $\Lambda$ must be positive semi-definite for Minkowskian signature (the upper sign), which corresponds to the upside-down potential generically. On the other hand, for Euclidean signature (the lower sign) $\Lambda$ must be negative semi-definite, and the system describes the usual harmonic oscillators. Similarly, the "signature" of $H_{\mathrm{DBI}}$, i.e. whether it corresponds to so $(1,1)$ or so $(2)$, is identical to that of the background geometry.

The isometry naturally gives rise to the Noether symmetry of the Dirac-Born-Infeld action. Taking care of the gauge fixing condition $s \equiv t=x^{0}$, one can see easily the Noether symmetry is

$$
\delta \phi=V^{t} \dot{\phi}-V^{\phi} .
$$

The corresponding Noether charge

$$
Q_{V}\left(\phi, p_{\phi}, t\right)=H_{\mathrm{DBI}}\left(\phi, p_{\phi}\right) V^{t}(t, \phi)-p_{\phi} V^{\phi}(t, \phi),
$$

generates the infinitesimal transformation via Poisson bracket, $\delta \phi=\left\{\phi, Q_{V}\right\}_{\text {P.B. }}$, and satisfies the conservation property,

$$
\left\{H_{\mathrm{DBI}}, Q_{V}\right\}_{\text {P.B. }}=\frac{\partial Q_{V}}{\partial t} .
$$

Due to the explicit time dependence of the Killing vector, $V^{t}(t, \phi), V^{\phi}(t, \phi)$, the right hand side does not vanish in general. The induced transformation of $X$ by $Q_{V}$ is

$$
\left\{X, Q_{V}\right\}_{\text {P.B. }}=V^{t} D_{t} X-V^{\phi} \mathcal{E}_{\phi} \mathcal{E}_{t}^{-2} .
$$

However, this does not give rise to a symmetry for the non-relativistic Lagrangian (3.10) or the DBI matrix model in (3.12), from the following reason. The Hamiltonian for the DBI matrix model is related to that of the DBI action by $H \equiv c \frac{1}{2} H_{\mathrm{DBI}}^{-2}$ (with $c$ some constant), so that $Q_{V}$ is not a symmetry generator for the Hamiltonian $H$

$$
\left\{H, Q_{V}\right\}_{\text {P.B. }}=-c H_{\mathrm{DBI}}^{-3} \partial_{t} Q_{V} \neq \partial_{t} Q_{V} .
$$

Nevertheless, we can modify the charge in order to satisfy the conservation,

$$
\mathcal{Q}_{V}:=Q_{V}+\sum_{n=1}^{\infty} \frac{t^{n}}{n !}\left(-1-c H_{\mathrm{DBI}}^{-3}\right)^{n} \frac{\partial^{n} Q_{V}}{\partial t^{n}}, \quad\left\{H, \mathcal{Q}_{V}\right\}_{\text {P.B. }}=\partial_{t} \mathcal{Q}_{V}
$$

This guarantees that the isometry of the background geometry is inherited by the matrix model as a Noether symmetry. Indeed, in [25] it was shown that matrix models with at most quadratic 
potential possess so(1,2) symmetry, and further allow for $\operatorname{osp}(1 \mid 2, \mathbf{R})$ supersymmetric extension. In Appendix $\mathrm{A}$, we review the $\mathrm{SO}(1,2)$ symmetry and present the explicit form of the transformations.

Finally, it is worth to note that the symmetry algebra for the corresponding matrix model is always so(1,2), while the isometry of the background can be different such as so(3) or Poincaré algebra. The so(1,2) symmetry in the matrix model may be identified with the global subalgebra $\mathrm{sl}(2, R)$ of the Virasoro algebra in string theory. Further, the quadratic matrix models possess a $W_{\infty}$ algebra $[25,36]$ which corresponds to the full Virasoro algebra. Considering the potentially limited applicability of the gravity action (2.2) to string theory, this might provide a criterium for true string vacua. In fact, if we define a new spatial coordinate $\sigma$ and a function $\Phi(\sigma)$ as

$$
\frac{\partial}{\partial \sigma}:=\frac{1}{\mathcal{E}_{\phi}(\phi)} \frac{\partial}{\partial \phi}, \quad \Phi(\sigma):=-\ln \mathcal{E}_{t}(\phi),
$$

then the defining equation of $\Lambda$ (3.21) can be rewritten as

$$
\frac{\partial^{2} \Phi}{\partial \sigma^{2}}= \pm \Lambda \exp (2 \Phi)
$$

Remarkably this coincides with the static equation of motion in Liouville field theory, provided $\Lambda$ is constant which is then identified as the Liouville background charge. This connection to the Liouville theory supports our approach to employ the Born-Infeld action for the backgrounds with constant $\Lambda$.

\section{Simple applications}

In this section we apply the above formalism, as simple exercises, to Rindler (2.18), anti-de-Sitter (2.19) and the linear dilaton (2.21) backgrounds. For the first two cases, we freeze the dilaton at the outer horizon, $\Phi \equiv \Phi\left(\phi_{h}^{\text {out }}\right)$, keeping only the leading order terms in the exact black hole solutions. Corrections to them are considered in the next section. All the resulting matrix models turn out to have potentials at most quadratic in $X$, and hence they have $\operatorname{SO}(1,2)$ symmetry and allow for the supersymmetric extension [25]. The corresponding geometries with the effective metric $e^{-2 \Phi} g_{\mu \nu}$ admit the isometry. 


\subsection{Rindler space}

Setting $g_{\hat{s}} \equiv e^{\Phi\left(\phi_{h}^{\text {out }}\right)}$, we get

$$
\Lambda=4 \pi^{2} T_{\mathrm{BH}}^{2}, \quad X=\frac{1}{4\left(\pi T_{\mathrm{BH}}\right)^{\frac{3}{2}} \sqrt{-u}},
$$

and hence, the DBI matrix model for the Rindler space reads

$$
\mathcal{L}_{\text {Rindler }}^{\text {DBI }}=m_{s} \operatorname{tr}\left[\frac{1}{2}\left(D_{t} X\right)^{2}+2 \pi^{2} T_{\mathrm{BH}}^{2} X^{2}\right] .
$$

Again, $X$ is non-negative, $X \geq 0$. The origin $X=0$ and the infinity $X=\infty$ correspond to the spatial infinity $u=-\infty$ and the horizon $u=0$, respectively. As follows from

$$
e^{\Phi(u)}=e^{\Phi\left(\phi_{h}^{\text {out }}\right)+\sqrt{2} u m_{s}}<e^{\Phi\left(\phi_{h}^{\text {out }}\right)},
$$

since $u$ is negative, the string coupling is bounded from above. This is in contrast to the linear dilaton background, where the string coupling diverges when $X \rightarrow \infty$, as we see below.

The matrix model corresponds to the first order approximation to the near-horizon D0dynamics for non-extremal black holes. The corrections to the potential coming from the higher orders as well as the contribution from the Chern-Simons sector are analyzed in detail in the next section. In any case, the leading order term in the potential near the horizon or for large $X$ is the above quadratic one. Accordingly D-branes fall with increasing velocity $D_{t} X \sim 2 \pi T_{\mathrm{BH}} X$ and the travel time is proportional to the logarithm of the distance with coefficient $T_{\mathrm{BH}}^{-1}$. For an external observer, they are falling forever (see also [6]) without reaching the horizon: a typical feature of black holes.

Since the coefficient of the quadratic potential is proportional to $T_{\mathrm{BH}}^{2}$, the energy spectra are given in the unit of the temperature. Thus, the corresponding partition function, $Z=\operatorname{Tr}\left(e^{-\beta_{\mathrm{BH}} E}\right)$ is independent of the temperature, as $\beta_{\mathrm{BH}} T_{\mathrm{BH}}=1$. Furthermore, the thermodynamical energy $\langle E\rangle=\operatorname{Tr}\left(e^{-\beta_{\mathrm{BH}} E} E\right) Z^{-1}$ is linear in the temperature, and the entropy becomes independent of the temperature,

$$
S=\ln Z+\beta_{\mathrm{BH}}\langle E\rangle, \quad \frac{\partial S}{\partial T_{\mathrm{BH}}}=0
$$

However, taking into account the next order corrections to the above matrix model for nonextremal black hole will make the entropy temperature dependent. 


\section{2 $A d S_{2}$ space: $A d S_{2} / \mathrm{CFT}_{1}$ correspondence}

Setting $g_{\hat{s}} \equiv e^{\Phi\left(\phi_{h}^{\text {out }}\right)}$ and from

$$
\Lambda=0, \quad m_{s} X=\frac{1}{\left(-u m_{s}\right)^{2}},
$$

the DBI matrix model for the $A d S_{2}$ space reads ( $c f .[27,47,48]$ )

$$
\mathcal{L}_{A d S_{2}}^{\mathrm{DBI}}=m_{s} \operatorname{tr}\left[\frac{1}{2}\left(D_{t} X\right)^{2}+512 m_{s} X\right]
$$

The string coupling is again bounded, as for the Rindler space (4.3).

This matrix model corresponds to the first order approximation to the near-horizon dynamics of D-particles for extremal black hole. For large $X$, the travel time is proportional to the square root of the distance, taking much longer than as in the non-extremal case above. Namely D-branes fall much slower in the extremal black hole. The matrix model possesses conformal symmetry [25], (A.11),

$$
\delta X=\delta t\left(D_{t} X-512 m_{s} t\right)-\frac{1}{2} \dot{\delta} t\left(X-256 m_{s} t^{2}\right), \quad \frac{\mathrm{d}^{3} \delta t}{\mathrm{~d} t^{3}}=0 .
$$

This corresponds to $\mathrm{SO}(1,2)$ symmetry and in particular, the choice $\delta t=t$ is the scale transformation. Consequently, the partition function is independent of the temperature, and the thermodynamical energy is zero. The entropy is again independent of the temperature (as in [49]).

\subsection{Linear dilaton background}

For the linear dilaton background (2.21), with the choice $g_{\hat{s}} \equiv g_{s}, \phi_{0} \equiv-\infty$, we obtain $\Lambda$ and $X$ (3.21), (3.6),

$$
\Lambda=\frac{2}{\alpha^{\prime}}, \quad X=\sqrt{\frac{\alpha^{\prime}}{2}} e^{\sqrt{2} \phi m_{s}} .
$$

Thus, the matrix model for the linear dilaton background reads $\left(\alpha^{\prime}=m_{s}^{-2}\right)$

$$
\mathcal{L}_{\text {L.D. }}=m_{s} \operatorname{tr}\left[\frac{1}{2}\left(D_{t} X\right)^{2}+\frac{1}{\alpha^{\prime}} X^{2}-\frac{1}{2} \mathcal{H}^{-2}\right] .
$$

Note that the contribution from the Chern-Simons action in (3.12) is trivial, i.e. just gives $-\frac{1}{2} \mathcal{H}^{-2}$. Eq.(4.8) implies that $X \geq 0$ and that $X$ is proportional to the string coupling. Since our analysis which uses the Born-Infeld action is valid only for small string coupling, the matrix model (4.9) 
is valid only for small $X$. The fact that $X \geq 0$ is similar to the $c=1$ matrix model, ${ }^{6}$ but different from the 0A matrix model [4], where $-\infty<X<+\infty$.

According to the equation of motion, the D-particles tend to move to the strongly coupled region, $X \rightarrow+\infty$ where they become lighter [37]. In the strong coupling region one should take into account the quantum correction to the gravity background, and the above matrix model may receive corrections at large $X$. This implies that the matrix model for the linear dilaton background is valid for small $X$.

To compare with the $c=1$ matrix model and the 0A matrix model, we note that the coefficient of the potential differs. In the $c=1$ matrix model the factor is $\frac{1}{2} \alpha^{\prime-1}$, while in the $0 \mathrm{~A}$ matrix model it is $\frac{1}{4} \alpha^{\prime-1}$. A rescaling of time may resolve this discrepancy. However, the normalization of the time coordinate is fixed by requiring $\left|g_{t t}\right| \rightarrow 1$ at spatial infinity. As we discussed before, the coefficient of the quadratic potential is independent of the choice of $g_{\hat{s}}$ in (3.6). In any case, the value of the coefficient given in (4.9) agrees with the conformal field theory result [4-6]. There the classical trajectory $X=\cosh \left(\sqrt{\frac{2}{\alpha^{\prime}}} t\right)$ was obtained from the peak of the quantum wave function. This trajectory satisfies the equation of motion of our matrix model (4.9), including the precise match of the frequency $\sqrt{\frac{2}{\alpha^{\prime}}}$.

\section{$5 \quad$ Matrix models for black holes}

In this section, we analyze in detail the matrix model for the non-extremal as well as the extremal black hole geometries. All the matrix models obtained in the following subsections [5.1-5.5] describe D-particles which fall towards the horizon of a black hole. The D-particles also represent fundamental strings. Since the region inside the horizon is causally disconnected, the matter falling towards the black hole - outside of it - provides an effective description of the black hole itself. Therefore, we identify the matrix models for D-particles in the black hole background with an effective description of the black hole itself. In principle, we could calculate thermodynamical properties of the black hole, e.g the entropy, from the matrix models. However this is beyond the scope of the present paper.

First, we ask if the exact black hole solution (2.16) admits any isometry for the effective metric

\footnotetext{
${ }^{6}$ For reviews of the $c=1$ matrix model see [38-40], and for the D-brane interpretation see [4,5, 28, 35, 41-46].
} 
$e^{-2 \Phi} g_{\mu \nu}$. We insert the solution into the expression for $\Lambda$ and get

$$
\left.\alpha^{\prime} \Lambda(\phi)\right|_{\text {exact }}=2+\frac{e^{2 \sqrt{2} \phi m_{s}}\left(g_{s} Q\right)^{2}}{128 \pi^{2}}\left[-64 \pi+e^{2 \sqrt{2} \phi m_{s}}\left(32 \pi \xi+\left(g_{s} Q\right)^{2}\left(1-4 \sqrt{2} \phi m_{s}\right)\right)\right],
$$

which is certainly not a constant in general. Hence, generic black holes do not admit an isometry. In the asymptotically flat region, $\phi \rightarrow-\infty, \Lambda$ converges to 2 , and the linear dilaton background naturally possesses an isometry.

For the genuine black hole solution, $\Lambda$ can be reexpressed as a function of the distance to the outer horizon, $u=\phi-\phi_{h}^{\text {out }} \leq 0$ and the temperature or $\varepsilon^{\text {out }}=-4 \pi \sqrt{\alpha^{\prime}} T_{\mathrm{BH}} \leq 0$, (2.14),

$$
\begin{aligned}
& \left.\alpha^{\prime} \Lambda(\phi)\right|_{\text {exact }} \\
& =2-2 \sqrt{2}\left(\varepsilon^{\text {out }}+2 \sqrt{2}\right) e^{2 \sqrt{2} u m_{s}}+\left(\varepsilon^{\text {out }}+2 \sqrt{2}\right)\left[\frac{1}{4}\left(\varepsilon^{\text {out }}+6 \sqrt{2}\right)-\sqrt{2}\left(\varepsilon^{\text {out }}+2 \sqrt{2}\right) u m_{s}\right] e^{4 \sqrt{2} u m_{s}} \\
& =\frac{1}{4}\left(\varepsilon^{\text {out }}\right)^{2}+\left(\varepsilon^{\text {out }}+2 \sqrt{2}\right)\left[-4 \varepsilon^{\text {out }}\left(u m_{s}\right)^{2}-\frac{32}{3}\left(\sqrt{2} \varepsilon^{\text {out }}+1\right)\left(u m_{s}\right)^{3}+\mathcal{O}\left[\left(u m_{s}\right)^{4}\right]\right] .
\end{aligned}
$$

Note that the linear term vanishes so that at the leading order, there should be an isometry, as seen in Sec. 4. In particular, for the extremal case, the zeroth order is trivial, indicating the absence of the quadratic potential as in (4.6).

A remarkable cancellation occurs when the black hole temperature saturates the Hagedorn temperature, or $\varepsilon^{\text {out }}=-2 \sqrt{2}$, as $\Lambda$ becomes exactly constant,

$$
\Lambda=\frac{2}{\alpha^{\prime}} \quad \text { if } \quad T_{\mathrm{BH}}=T_{\text {Hag. }},
$$

which implies an isometry of the background and hence a quadratic potential in the corresponding matrix model, as we will see shortly.

For the exact black hole solutions, we aim to calculate the matrix coordinate $X$ as a function of the Liouville coordinate $\phi$, or the distance from the horizon, $u=\phi-\phi_{h}^{\text {out }}$. We focus on the region outside of the outer horizon so that $u \leq 0$ and $\varepsilon^{\text {out }}<0$. We obtain

$$
X=\frac{g_{s}}{g_{\hat{s}}} \int_{\phi_{0}}^{\phi} \mathrm{d} \phi^{\prime} \frac{e^{\sqrt{2} \phi^{\prime} m_{s}}}{l\left(\phi^{\prime}\right)^{\frac{3}{2}}}=\frac{g_{s} e^{\sqrt{2} \phi_{h}^{\text {out }} m_{s}}}{g_{\hat{s}} m_{s}} \int_{u_{0} m_{s}}^{u m_{s}} \mathrm{~d} y \frac{e^{-2 \sqrt{2} y}}{\left[\left(\varepsilon^{\text {out }}+2 \sqrt{2}\right) y-1+e^{-2 \sqrt{2} y}\right]^{\frac{3}{2}}} .
$$

Note that $\phi \leq \phi_{h}^{\text {out }}$ and $y \leq 0$. Putting $\phi_{0} \equiv-\infty$, we have $0<X<\infty$ such that $X=\infty$ corresponds to the outer horizon $\phi=\phi_{h}^{\text {out }}$, while $X=0$ corresponds to the asymptotically flat spatial infinity, $\phi=-\infty$. Roughly, the matrix coordinate $X$ stretches the near-horizon region 
infinitely. However, as we do not know the exact expression for the integral, except the Hagedorn temperature case, i.e. $\varepsilon^{\text {out }}=-2 \sqrt{2}$, we will power expand the integrand in $y$ and perform the indefinite integral order by order. This procedure can be regarded as our regularization scheme for $X$, as we make any integration constant trivial. ${ }^{7}$

It is now natural to fix the arbitrary parameter as

$$
g_{\hat{s}} \equiv g_{s} e^{\sqrt{2} \phi_{h}^{\text {out }} m_{s}}=e^{\Phi\left(\phi_{h}^{\text {out }}\right)}=4 \pi Q^{-1} \sqrt{\sqrt{2}\left(T_{\text {Hag. }}-T_{\mathrm{BH}}\right)},
$$

which is the string coupling at the horizon, the region we are interested in. Consequently we have an expression for the black hole temperature in terms of $g_{\hat{s}} Q$

$$
T_{\mathrm{BH}}=\frac{m_{s}}{4 \pi}\left|\varepsilon^{\mathrm{out}}\right|=\frac{m_{s}}{\sqrt{2} \pi}\left(1-\frac{\left(g_{s} Q\right)^{2}}{16 \pi} e^{2 \sqrt{2} \phi_{h}^{\text {out }} m_{s}}\right)=\frac{m_{s}}{\sqrt{2} \pi}\left(1-\frac{\left(g_{\hat{s}} Q\right)^{2}}{16 \pi}\right)>0 .
$$

Further, the DBI potential is

$$
-\frac{1}{2 g_{\hat{s}}^{2} \mathcal{E}_{t}^{2}}=\frac{1}{2-8 \pi\left(T_{\text {Hag. }}-T_{\mathrm{BH}}\right) u-2 e^{-2 \sqrt{2} u m_{s}}},
$$

while the CS potential is, with the gauge choice, $C_{t}\left(\phi_{h}^{\text {out }}\right) \equiv 0$,

$$
\frac{1}{2\left(\mathcal{H}+g_{\hat{s}} m_{s}^{-1} C_{t}\right)^{2}}=\frac{1}{2\left(\mathcal{H}+\sqrt{8 \sqrt{2} m_{s}^{-1}\left(T_{\text {Hag. }}-T_{\mathrm{BH}}\right)}\left(-u m_{s}\right)\right)^{2}} .
$$

We recall (2.17) that unlike in the linear dilaton background, the string coupling in the black hole background is bounded from above by the inverse of the flux

$$
e^{\Phi\left(\phi^{\text {out }}\right)} \leq \frac{4 \sqrt{\pi}}{Q}
$$

Thus, by taking large $Q$, the string coupling can be kept small and quantum corrections can be neglected. The corresponding matrix model for the black hole then can be trusted over the entire

${ }^{7}$ An alternative expansion by $e^{u m_{s}}$ is available for the asymptotic region $u \rightarrow-\infty$,

$$
m_{s} X=\sum_{n=0}^{\infty} \frac{(2 n+1) !}{\sqrt{2}(n !)^{2}}(-4 \sqrt{2} \Delta)^{-n} e^{\sqrt{2}(2 n+1) \Delta} \int_{-\infty}^{\sqrt{2}\left(u m_{s}-\Delta\right)} \mathrm{d} \eta \eta^{n} e^{(2 n+1) \eta},
$$

where we adopted (5.5) and set $\Delta \equiv\left(\varepsilon^{\text {out }}+2 \sqrt{2}\right)^{-1}$. The leading term $n=0$ corresponds to (4.8) and leads to the matrix model for the linear dilaton background. The higher order terms then give the corrections. However, in the present paper we focus on the near-horizon region, and do not pursue the analysis of this expansion. 
region up to $0<X<\infty$.

Using the chain rule (3.35), the differentiation of the DBI potential reads

$$
\frac{\mathrm{d}}{\mathrm{d} X}\left(-\frac{1}{2 g_{\hat{s}}^{2} \mathcal{E}_{t}(\phi(X))^{2}}\right)=-2 \pi \sqrt{\left|g_{t t}\right|} e^{\sqrt{2} u m_{s}}\left[\frac{\mathcal{T}(u)}{\left|g_{t t}\right|}+T_{\text {Hag. }}\right],
$$

where we set

$$
\mathcal{T}(u) \equiv \frac{1}{4 \pi} \frac{\mathrm{d} g_{t t}(\phi)}{\mathrm{d} \phi}=\left[T_{\mathrm{BH}}-2 \sqrt{2}\left(T_{\mathrm{Hag} .}-T_{\mathrm{BH}}\right) u m_{s}\right] e^{2 \sqrt{2} u m_{s}} .
$$

Outside the outer horizon, $u$ is negative so that $\mathcal{T}(u)$ is positive. Thus the right hand side of (5.10) is negative, and there is no extremal point of the DBI potential outside the outer horizon, except at the spatial infinity, $X=0$ or $u=-\infty$. The DBI potential is monotonically decreasing over the entire range, from zero at the spatial infinity to $-\infty$ at the outer horizon.

\subsection{String/Black hole transition at the Hagedorn temperature: Background independence in the vanishing string coupling limit}

At the Hagedorn temperature, where $\varepsilon^{\text {out }}=-2 \sqrt{2}$, from (5.8) the CS matrix model becomes trivial, i.e. just gives the constant term $-\frac{1}{2} \mathcal{H}^{-2}$. The integration for $X$ (5.4) can be exactly performed, with $u_{0} \equiv-\infty$,

$$
m_{s} X=\left(2 e^{-2 \sqrt{2} u m_{s}}-2\right)^{-\frac{1}{2}}
$$

and hence,

$$
u m_{s}=-\frac{\sqrt{2}}{4} \ln \left[1+\frac{1}{2\left(m_{s} X\right)^{2}}\right] .
$$

Finally substituting this into (3.12), we obtain the matrix model for the black hole at the Hagedorn temperature,

$$
\mathcal{L}_{\text {Black Hole }}^{\text {Hagedorn }}=m_{s} \operatorname{tr}\left[\frac{1}{2}\left(D_{t} X\right)^{2}+\frac{1}{\alpha^{\prime}} X^{2}-\frac{1}{2} \mathcal{H}^{-2}\right],
$$

with $\mathcal{H}$ as in (3.15) and $\alpha^{\prime}=m_{s}^{-2}$. We observe that the resulting matrix model is identical to the one for the linear dilaton background (4.9). This is a nontrivial result, since the corresponding geometries (2.21) and (2.20) are distinct. Moreover, the expressions for the matrix variable in terms of the Liouville coordinate (4.8) and (5.12) are different.

Note again that we interpret the matrix model (5.14) as an effective description of the black hole, as discussed on page 20. Hence, we may view the fact that (3.12) reduces to (5.14) at the Hagedorn temperature as realization of the string/black hole transition [7-15]. From 
$T_{\text {Hag. }}-T_{\mathrm{BH}}=\frac{m_{s}}{16 \sqrt{2} \pi^{2}}\left(g_{\hat{s}} Q\right)^{2}$, the transition occurs when $g_{\hat{s}} Q$ vanishes, i.e. in the weak coupling limit.

What we observe here is that when the string coupling vanishes, there is a degeneracy of the matrix models for different geometries, (4.9) and (5.14), i.e. the matrix models for the linear dilaton background and for the black hole background at the Hagedorn temperature. Moreover when the string coupling goes to zero, the closed string modes decouple, as the closed string coupling is the square of the open string coupling. This implies that the dynamics of the Dparticles, which correspond to open strings, becomes independent of the background geometry. Hence we conjecture that in the vanishing string coupling limit, the D0-brane dynamics or string theory itself becomes independent of the background geometry and the resulting matrix model is of universal form, given by the above matrix model (5.14). This is consistent with universality in a thermodynamical system at a critical point (see e.g. [50]).

\subsection{Near the Hagedorn temperature}

Taking $T_{\text {Hag. }}-T_{\mathrm{BH}}=\frac{m_{s}}{4 \pi}\left(\varepsilon^{\text {out }}+2 \sqrt{2}\right)$ as a small parameter, we analyze the leading order correction to (5.14). First we expand the integrand (5.4) up to the linear order,

$$
\frac{e^{-2 \sqrt{2} y}}{\left[\left(\varepsilon^{\text {out }}+2 \sqrt{2}\right) y-1+e^{-2 \sqrt{2} y}\right]^{\frac{3}{2}}} \simeq \frac{e^{-2 \sqrt{2} y}}{\left[-1+e^{-2 \sqrt{2} y}\right]^{\frac{3}{2}}}-\frac{3 y e^{-2 \sqrt{2} y}}{2\left[-1+e^{-2 \sqrt{2} y}\right]^{\frac{5}{2}}}\left(\varepsilon^{\text {out }}+2 \sqrt{2}\right) .
$$

In this subsection, ' $\simeq$ ' denotes equality up to linear order in $\left(\varepsilon^{\text {out }}+2 \sqrt{2}\right)$. It is convenient to introduce

$$
W:=e^{-2 \sqrt{2} u m_{s}}-1 .
$$

The matrix coordinate $X$ is, after integration from $u_{0} \equiv-\infty$,

$$
m_{s} X \simeq \frac{1}{\sqrt{2}} W^{-\frac{1}{2}}+\left[\frac{1}{4} W^{-\frac{1}{2}}-\frac{\sqrt{2}}{4} u m_{s} W^{-\frac{3}{2}}-\frac{1}{4} \operatorname{arccot}\left(W^{\frac{1}{2}}\right)\right]\left(\varepsilon^{\text {out }}+2 \sqrt{2}\right) .
$$

It follows that the inverse function reads

$$
\begin{aligned}
-u m_{s} \simeq & \frac{\sqrt{2}}{4} \ln \left[1+\frac{1}{2\left(m_{s} X\right)^{2}}\right] \\
& +\left[\frac{2 m_{s} X+2\left(m_{s} X\right)^{3} \ln \left(1+\frac{1}{2\left(m_{s} X\right)^{2}}\right)-\sqrt{2} \arctan \left(\sqrt{2} m_{s} X\right)}{8 m_{s} X+16\left(m_{s} X\right)^{3}}\right]\left(\varepsilon^{\text {out }}+2 \sqrt{2}\right) .
\end{aligned}
$$


The DBI potential is then

$$
-\frac{1}{2 g_{\hat{s}}^{2} \mathcal{E}_{t}^{2}} \simeq-\left(m_{s} X\right)^{2}+\left[\frac{1}{\sqrt{2}}\left(m_{s} X\right)^{2}-\frac{1}{2}\left(m_{s} X\right) \arctan \left(\sqrt{2} m_{s} X\right)\right]\left(\varepsilon^{\text {out }}+2 \sqrt{2}\right),
$$

while for the CS part, due to the presence of the factor $\sqrt{\varepsilon^{\text {out }}+2 \sqrt{2}}$ in (5.8), only the zeroth order in (5.18) contributes.

All together, the matrix model near the Hagedorn temperature is given by

$$
\mathcal{L}_{\text {Near Hagedorn }}=\mathcal{L}_{\text {Near Hag. }}^{\text {DBI }}+\mathcal{L}_{\text {Near Hag. }}^{\text {CS }},
$$

where

$$
\begin{aligned}
& \mathcal{L}_{\text {Near Hag. }}^{\text {DBI }}=m_{s} \operatorname{tr}\left[\frac{1}{2}\left(D_{t} X\right)^{2}+\frac{1}{\alpha^{\prime}} X^{2}+2 \pi\left(T_{\text {Hag. }}-T_{\mathrm{BH}}\right) X\left[\arctan \left(\sqrt{2} m_{s} X\right)-\sqrt{2} m_{s} X\right]\right], \\
& \mathcal{L}_{\text {Near Hag. }}^{\text {CS }}=m_{s} \operatorname{tr}\left[-\frac{2}{3}\left[\sqrt{\sqrt{2} m_{s}^{-1}\left(T_{\text {Hag. }}-T_{\mathrm{BH}}\right)} \ln \left(1+\frac{1}{2\left(m_{s} X\right)^{2}}\right)-\frac{1}{3} \mathcal{H}\right]^{2} \mathcal{H}^{-4}-\frac{23}{54} \mathcal{H}^{-2}\right] .
\end{aligned}
$$

We recall that there are two types of D-particles and of $\mathrm{RR}$ flux. $\mathrm{D} 0^{(+)}$are charged under $C^{(+)}$ only, and $\mathrm{D0}^{(-)}$under $C^{(-)}$. The above Chern-Simons part is valid for the charged D-particles. See the discussion before (3.16).

\subsection{Non-extremal black hole}

Here we calculate $X$ for the generic non-extremal black hole geometry. The integrand in (5.4) has the following power expansion in $y<0$, with $\varepsilon^{\text {out }}<0$,

$$
\begin{aligned}
& \frac{e^{\sqrt{2} y}}{\left[1+\left(\left(\varepsilon^{\text {out }}+2 \sqrt{2}\right) y-1\right) e^{2 \sqrt{2} y]^{\frac{3}{2}}}\right.} \\
& =\frac{1}{\left|\varepsilon^{\text {out }}\right|^{\frac{3}{2}}(-y)^{\frac{3}{2}}}+\frac{2\left(\sqrt{2}\left|\varepsilon^{\text {out }}\right|-3\right)}{\left|\varepsilon^{\text {out }}\right|^{\frac{5}{2}}(-y)^{\frac{1}{2}}}+\frac{\left(4\left|\varepsilon^{\text {out }}\right|^{2}-16 \sqrt{2}\left|\varepsilon^{\text {out }}\right|+30\right)}{\left|\varepsilon^{\text {out }}\right|^{\frac{7}{2}}}(-y)^{\frac{1}{2}}+\mathcal{O}\left[(-y)^{\frac{3}{2}}\right],
\end{aligned}
$$

and hence, after indefinite integration, $X$ reads in terms of $u=\phi-\phi_{h}^{\text {out }}<0$,

$$
m_{s} X \simeq \frac{2\left|\varepsilon^{\text {out }}\right|^{-\frac{3}{2}}}{\sqrt{-u m_{s}}}-\frac{4\left(\sqrt{2}\left|\varepsilon^{\text {out }}\right|-3\right)}{\left|\varepsilon^{\text {out }}\right|^{\frac{5}{2}}} \sqrt{-u m_{s}}-\frac{4\left(2\left|\varepsilon^{\text {out }}\right|^{2}-8 \sqrt{2}\left|\varepsilon^{\text {out }}\right|+15\right)}{3\left|\varepsilon^{\text {out }}\right|^{\frac{7}{2}}}\left(-u m_{s}\right)^{\frac{3}{2}}
$$


Here $\varepsilon^{\text {out }}$ as given by (5.6) is related to the temperature of the black hole, $y$ is the integration variable of (5.4), and $m_{s}$ is the string mass, $m_{s}=\alpha^{\prime-\frac{1}{2}}$.

Similarly, up to terms linear in $\left(-u m_{s}\right)$, we have

$$
\begin{aligned}
& \frac{1}{\left(m_{s} X\right)^{2}} \simeq \frac{\left|\varepsilon^{\text {out }}\right|^{3}\left(-u m_{s}\right)}{4}, \\
& \left(m_{s} X\right)^{2} \simeq \frac{4}{\left|\varepsilon^{\text {out }}\right|^{3}\left(-u m_{s}\right)}-\frac{16\left(\sqrt{2}\left|\varepsilon^{\text {out }}\right|-3\right)}{\left|\varepsilon^{\text {out }}\right|^{4}}+\frac{32\left(2\left|\varepsilon^{\text {out }}\right|^{2}-5 \sqrt{2}\left|\varepsilon^{\text {out }}\right|+6\right)}{3\left|\varepsilon^{\text {out }}\right|^{5}}\left(-u m_{s}\right), \\
& \left(m_{s} X\right)^{4} \simeq \frac{16}{\left|\varepsilon^{\text {out }}\right|^{6}\left(-u m_{s}\right)^{2}}-\frac{128\left(\sqrt{2}\left|\varepsilon^{\text {out }}\right|-3\right)}{\left|\varepsilon^{\text {out }}\right|^{7}\left(-u m_{s}\right)}+\frac{256\left(8\left|\varepsilon^{\text {out }}\right|^{2}-23 \sqrt{2}\left|\varepsilon^{\text {out }}\right|+33\right)}{3\left|\varepsilon^{\text {out }}\right|^{8}} \\
& \quad-\frac{256\left(32 \sqrt{2}\left|\varepsilon^{\text {out }}\right|^{3}-243\left|\varepsilon^{\text {out }}\right|^{2}+300 \sqrt{2}\left|\varepsilon^{\text {out }}\right|-240\right)}{15\left|\varepsilon^{\text {out }}\right|^{9}}\left(-u m_{s}\right) .
\end{aligned}
$$

For the analysis of the non-extremal black hole in this subsection, we now take the near-horizon limit by neglecting all the powers higher than one:

$$
\left(-u m_{s}\right)^{n} \cong 0, \quad n>1
$$

In other words, we focus on length scales up to the string scale, ${ }^{8}|u|<\frac{1}{N_{\text {cut }}} \sqrt{\alpha^{\prime}}$, where the cutoff is controlled by a natural number $N_{\text {cut }}$ of order one. ${ }^{9}$ Therefore the expansion is valid for $X$ in the range

$$
X \gtrsim X_{\min } \equiv \frac{\sqrt{m_{s} N_{\mathrm{cut}}}}{4\left(\pi T_{\mathrm{BH}}\right)^{\frac{3}{2}}} .
$$

\footnotetext{
${ }^{8}$ Alternatively, one may neglect all the positive powers restricting on the region $|u| \ll \sqrt{\alpha^{\prime}}$, but it will miss all the negative powers of $X$ as well as the Chern-Simons part, and eventually reduce to the matrix model for the Rindler space (4.2).

${ }^{9}$ Note that unlike in higher dimensions, in the two-dimensional near-horizon limit, $\alpha^{\prime}$ may remain finite.
} 
From (5.24), we can now solve for $\left(-u m_{s}\right),\left(-u m_{s}\right)^{-1}$ and $\left(-u m_{s}\right)^{-2}$ in terms of $X$,

$$
\begin{aligned}
&\left(-u m_{s}\right) \cong \frac{4}{\left|\varepsilon^{\text {out }}\right|^{3}\left(m_{s} X\right)^{2}}, \\
& \frac{1}{\left(-u m_{s}\right)} \cong \frac{\left|\varepsilon^{\text {out }}\right|^{3}}{4}\left(m_{s} X\right)^{2}+\frac{4\left(\sqrt{2}\left|\varepsilon^{\text {out }}\right|-3\right)}{\left|\varepsilon^{\text {out }}\right|}-\frac{32\left(2\left|\varepsilon^{\text {out }}\right|^{2}-5 \sqrt{2}\left|\varepsilon^{\text {out }}\right|+6\right)}{3\left|\varepsilon^{\text {out }}\right|^{5}\left(m_{s} X\right)^{2}}, \\
& \frac{1}{\left(-u m_{s}\right)^{2}} \cong \frac{\left|\varepsilon^{\text {out }}\right|^{6}}{16}\left(m_{s} X\right)^{4}+2\left(\sqrt{2}\left|\varepsilon^{\text {out }}\right|-3\right)\left[\left|\varepsilon^{\text {out }}\right|^{2}\left(m_{s} X\right)^{2}+\frac{8\left(2 \sqrt{2}\left|\varepsilon^{\text {out }}\right|-7\right)}{3\left|\varepsilon^{\text {out }}\right|^{2}}\right] \\
& \quad-\frac{64\left(\left|\varepsilon^{\text {out }}\right|-2 \sqrt{2}\right)\left(8 \sqrt{2}\left|\varepsilon^{\text {out }}\right|^{2}-45\left|\varepsilon^{\text {out }}\right|+30 \sqrt{2}\right)}{15\left|\varepsilon^{\text {out }}\right|^{6}\left(m_{s} X\right)^{2}} .
\end{aligned}
$$

Accordingly we have for the DBI potential,

$$
\begin{aligned}
\frac{1}{g_{\hat{s}}^{2} \mathcal{E}_{t}^{2}} & =e^{2 \sqrt{2} u m_{s}}\left[1-\left(1+\left(\left|\varepsilon^{\text {out }}\right|-2 \sqrt{2}\right) u m_{s}\right) e^{2 \sqrt{2} u m_{s}}\right]^{-1} \\
& \cong \frac{1}{\left|\varepsilon^{\text {out }}\right|\left(-u m_{s}\right)}-\frac{4}{\left|\varepsilon^{\text {out }}\right|^{2}}-\frac{8\left(\frac{\sqrt{2}}{3}\left|\varepsilon^{\text {out }}\right|-2\right)}{\left|\varepsilon^{\text {out }}\right|^{3}}\left(-u m_{s}\right) \\
& \cong \frac{\left|\varepsilon^{\text {out }}\right|^{2}}{4}\left(m_{s} X\right)^{2}+\frac{4 \sqrt{2}\left(\left|\varepsilon^{\text {out }}\right|-2 \sqrt{2}\right)}{\left|\varepsilon^{\text {out }}\right|^{2}}-\frac{64\left(\left|\varepsilon^{\text {out }}\right|-2 \sqrt{2}\right)}{3\left|\varepsilon^{\text {out }}\right|^{5}\left(m_{s} X\right)^{2}}
\end{aligned}
$$

From (2.15) and (5.6), $\left|\varepsilon^{\text {out }}\right|$ corresponds to the black hole temperature and $2 \sqrt{2}$ to the Hagedorn temperature.

The analysis of the near-horizon limit of the CS matrix model is a bit more subtle due to the arbitrary parameter $\mathcal{H}$. From (5.8), there is a critical value of $\mathcal{H}$, depending on the temperature and the cutoff,

$$
\mathcal{H}_{\mathrm{c}}\left(T_{\mathrm{BH}}\right) \equiv \frac{1}{N_{\text {cut }}} \sqrt{8 \sqrt{2} m_{s}^{-1}\left(T_{\text {Hag. }}-T_{\mathrm{BH}}\right)} .
$$

When $|\mathcal{H}|>\mathcal{H}_{\mathrm{c}}$, the CS matrix model has a regular expansion in $u m_{s}$ and in the near-horizon region (5.25), we need to keep only the linear term, leading to a $X^{-2}$ potential from (5.27). On the other hand, when $0<|\mathcal{H}| \leq \mathcal{H}_{\mathrm{c}}$, the expansion depends on whether $\left|u m_{s}\right|$ is bigger or smaller than $|\mathcal{H}|$. Therefore there is no closed expression for the matrix model, valid in the entire near-horizon region. Hence we exclude the parameter range $0<|\mathcal{H}| \leq \mathcal{H}_{\mathrm{c}}$, as far as the Chern-Simons part is 
concerned. Finally when $\mathcal{H}=0$, from (15.8) the CS potential is of the form $\left(u m_{s}\right)^{-2}$. From (5.27) this gives rise to a potential involving $X^{4}, X^{2}$ and $X^{-2}$ terms.

All together, the matrix model for the D-particles in the near-horizon region of the nonextremal black hole reads

$$
\mathcal{L}_{\text {Black Hole }}=\mathcal{L}_{\text {Black Hole }}^{\text {DBI }}+\mathcal{L}_{\text {Black Hole }}^{\text {CS }}
$$

where

$$
\begin{aligned}
& \mathcal{L}_{\text {Black Hole }}^{\text {DBI }}=m_{s} \operatorname{tr}\left[\frac{1}{2}\left(D_{t} X\right)^{2}+2 \pi^{2} T_{\mathrm{BH}}^{2} X^{2}+\left(\frac{\left(T_{\text {Hag. }}-T_{\mathrm{BH}}\right) m_{s}^{2}}{24 \pi^{4} T_{\mathrm{BH}}^{5}}\right) \frac{1}{X^{2}}-\frac{\left(T_{\text {Hag. }}-T_{\mathrm{BH}}\right) m_{s}}{\sqrt{2} \pi T_{\mathrm{BH}}^{2}}\right], \\
& \mathcal{L}_{\text {Black Hole }}^{\mathrm{CS}}=m_{s} \operatorname{tr}\left[\frac{\sqrt{2 \sqrt{2} m_{s}\left(T_{\text {Hag. }}-T_{\mathrm{BH}}\right)}}{8 \pi^{3} \mathcal{H}^{3} T_{\mathrm{BH}}^{3} X^{2}}-\frac{1}{2 \mathcal{H}^{2}}\right] .
\end{aligned}
$$

Compared to the matrix model for Rindler space (4.2), the above DBI matrix model contains an additional $X^{-2}$ contribution to the potential. In fact, the inverse square potential was proposed by Jevicki and Yoneya for the matrix description of a two-dimensional black hole, the so-called deformed matrix model [26].

A few other comments are in order: The matrix model is valid over the range $X_{\min }<X$ (5.26), and $\mathcal{H}_{\mathrm{c}}<|\mathcal{H}|$ (5.29) for the CS sector. If $\mathcal{H}$ vanishes, the CS potential can be read off from (5.8). The $X^{-2}$ repulsive potential in the DBI matrix model has an analogy with the known relativistic "centrifugal" barrier, which is dominant far from the horizon but suppressed by the gravitational attraction $X^{2}$ near the horizon (see e.g. [10]). On the other hand, the inverse square potential in the CS matrix model can be either repulsive or attractive depending on the sign of $\mathcal{H}, \mathcal{H}_{\mathrm{c}}<\mathcal{H}$ or $\mathcal{H}<-\mathcal{H}_{\mathrm{c}}$ respectively. At the Hagedorn temperature the above matrix model again reduces to the matrix model for the linear dilaton background (4.9). Hence our small $u m_{s}$ calculation of this subsection is consistent with the exact result of Sec. [5.1]

Combining the DBI and CS parts, the total potential is

$$
V_{\text {total }}=m_{s} \operatorname{tr}\left[-2 \pi^{2} T_{\mathrm{BH}}^{2} X^{2}-\left(\frac{\left(T_{\mathrm{Hag}}-T_{\mathrm{BH}}\right) m_{s}^{2}}{24 \pi^{4} T_{\mathrm{BH}}^{5}}+\frac{\sqrt{2 \sqrt{2} m_{s}\left(T_{\mathrm{Hag}}-T_{\mathrm{BH}}\right)}}{8 \pi^{3} \mathcal{H}^{3} T_{\mathrm{BH}}^{3}}\right) \frac{1}{X^{2}}\right],
$$

so that depending on the value of $\mathcal{H}$, the potential develops an infinite wall or cliff near the origin. The former will surely prevent the D-particles from approaching the forbidden region, 
$0 \leq X<X_{\min }$, and the matrix model can be trusted over the entire region $0 \leq X \leq \infty$. Explicitly, this is the case when $\mathcal{H}$ is negative and satisfies

$$
\mathcal{H}_{\mathrm{c}}<-\mathcal{H}<\left(\frac{18 \sqrt{2} \pi^{2} T_{\mathrm{BH}}^{4}}{m_{s}^{3}\left(T_{\text {Hag. }}-T_{\mathrm{BH}}\right)}\right)^{\frac{1}{6}} .
$$

From (5.29) the consistency requires that

$$
\frac{T_{\text {Hag. }}}{1+\sqrt{3 \pi}\left(\frac{1}{8} N_{\text {cut }}\right)^{\frac{3}{2}}}<T_{\text {BH }} \leq T_{\text {Hag. }} .
$$

In particular, the above condition can always be satisfied near the Hagedorn temperature, where $g_{\hat{s}} Q$ is small. In this case, the matrix model becomes

$$
\mathcal{L}_{\text {Black Hole }} \simeq m_{s} \operatorname{tr}\left[\frac{1}{2}\left(D_{t} X\right)^{2}+\frac{1}{\alpha^{\prime}} X^{2}+\alpha^{\prime}\left(1+\frac{12}{\mathcal{H}^{3} g_{\hat{s}} Q}\right) \frac{\left(g_{\hat{s}} Q\right)^{2}}{96 \pi X^{2}}\right],
$$

which agrees with (5.21), up to a shift of $X$ since we performed an indefinite integration for (5.22).

The Fermi sea level is $E_{\mathrm{FS}}=-\frac{1}{16 \pi}\left(g_{\hat{s}} Q\right)^{2}-\frac{1}{2} \mathcal{H}^{-2}$. With a suitable negative choice of $\mathcal{H}$, such that $\mathcal{H}^{-3} \propto-g_{\hat{s}} Q$, this Lagrangian can be identified with the $0 \mathrm{~A}$ matrix model proposed in $[4,45]$.

\subsection{Extremal black hole}

For the extremal black hole we have $\varepsilon=0$, and the integrand in (5.4) has a regular pole. Consequently $X$ has an expansion different from (5.23),

$$
X=\frac{\sqrt{\pi}}{4 g_{\hat{s}} m_{s} Q}\left[\frac{1}{\left(-u m_{s}\right)^{2}}+\frac{2 \sqrt{2}}{\left(-u m_{s}\right)}-\frac{4 \ln \left(-u m_{s}\right)}{3}+\frac{8 \sqrt{2}\left(-u m_{s}\right)}{135}+\mathcal{O}\left[\left(-u m_{s}\right)^{2}\right]\right] .
$$

We take the near-horizon limit by neglecting all the terms which vanish when $u m_{s} \rightarrow 0$ or

$$
\left(-u m_{s}\right)^{n} \cong 0, \quad\left(-u m_{s}\right)^{n} \ln \left(-u m_{s}\right) \cong 0, \quad n>0 .
$$

Accordingly, after setting

$$
g_{\hat{s}} \equiv e^{\Phi\left(\phi_{h}^{\text {out }}\right)}=4 \sqrt{\pi} Q^{-1} \propto g_{s},
$$

we get

$$
4 \sqrt{m_{s} X} \cong \frac{1}{\left(-u m_{s}\right)}+\sqrt{2}, \quad \ln \left(16 m_{s} X\right) \cong-2 \ln \left(u m_{s}\right)
$$


so that with (5.37)

$$
\left(-u m_{s}\right)^{-2} \cong 16 m_{s} X-8 \sqrt{2 m_{s} X}-\frac{2}{3} \ln \left(16 m_{s} X\right)+4 .
$$

The DBI potential becomes

$$
\begin{aligned}
-\frac{1}{2 g_{\hat{s}}^{2} \mathcal{E}_{t}(\phi(X))^{2}} & \cong-32\left(-u m_{s}\right)^{-2}-\frac{64 \sqrt{2}}{3}\left(-u m_{s}\right)^{-1}-\frac{64}{9} \\
& \cong-512 m_{s} X+\frac{512}{3} \sqrt{2 m_{s} X}+\frac{64}{3} \ln \left(16 m_{s} X\right)-\frac{832}{9} .
\end{aligned}
$$

On the other hand for the CS potential we have $\left(\mathcal{H}+g_{\hat{s}} C_{t} m_{s}^{-1}\right)^{-2}=\left(\mathcal{H}+\sqrt{\frac{8}{\pi}}\left|u m_{s}\right|\right)^{-2}$, which has a regular expansion for $|\mathcal{H}|>\sqrt{\frac{8}{\pi}}\left|u m_{s}\right|$, and hence becomes trivial in the near-horizon limit (5.38). On the other hand, the near-horizon limit justifies us to focus on the parameter range $|\mathcal{H}|>\sqrt{\frac{8}{\pi}}\left|u m_{s}\right|$ or $\mathcal{H}=0$, as done for the non-extremal case. When $\mathcal{H}=0$, we have $\left(-u m_{s}\right)^{-2}$ for the CS potential of which the expression can be read off from (5.41). For large $|\mathcal{H}|$ we conclude that the matrix model for D-particles in the near-horizon region of the extremal black hole is of the form

$$
\mathcal{L}_{\text {extr. B.H. }}=\operatorname{tr}\left[\frac{1}{2}\left(D_{t} X\right)^{2}+512 m_{s} X-\frac{512}{3} \sqrt{2 m_{s} X}-\frac{64}{3} \ln \left(16 m_{s} X\right)+\frac{832}{9}-\frac{1}{2} \mathcal{H}^{-2}\right] .
$$

The leading order linear contribution to the potential corresponds to the exact $A d S_{2}$ background (4.6), while the other give corrections necessary for the extremal black hole. The correction terms break the conformal symmetry and the partition function is no longer temperature independent. It will be interesting to calculate the entropy of the above matrix model and to compare with the gravity result. 


\subsection{Conformal field theory background}

Here we consider the black hole background in a coset conformal field theory at level $\kappa(2.22)$

$$
\mathrm{d} s^{2}=\kappa \alpha^{\prime}\left(-\tanh ^{2} \rho \mathrm{d} \tau^{2}+\mathrm{d} \rho^{2}\right), \quad \Phi=-\ln \cosh \rho+\Phi_{h} .
$$

When $\kappa=1 / 2$, this geometry coincides with the black hole at the Hagedorn temperature (2.20), as shown in (2.24), while for generic $\kappa \neq 1 / 2$, it is not a classical solution of the gravity action (2.2). The corresponding matrix model is then

$$
\mathcal{L}_{\kappa \text { Black Hole }}=m_{s} \operatorname{tr}\left[\frac{1}{2}\left(D_{t} X\right)^{2}+\frac{1}{2 \kappa \alpha^{\prime}} X^{2}-\frac{1}{2} \mathcal{H}^{-2}\right]
$$

This coincides with the matrix model for the Rindler space (4.2) if we identify the black hole temperature with

$$
T_{\mathrm{BH}}=\frac{1}{2 \pi \sqrt{\kappa \alpha^{\prime}}} .
$$

This agrees with the conformal field theory analysis [13]. Especially, when $\kappa=1 / 2$, we have precisely the same matrix model as for the black hole at the Hagedorn temperature (5.14). As was discussed in Sec 5.1 this also coincides with the matrix model for the linear dilaton background. Our interpretation of this result as the black hole/string transition agrees with the conformal field theory analysis [13], where $\kappa=1 / 2$ was shown to correspond to the far extreme of the string phase.

\section{Acknowledgments}

We thank Johannes Große and Dimitrios Tsimpis for helpful discussions. JHP wishes to thank Tadashi Takayanagi for useful electronic correspondence. JHP and CS are grateful to the Alexander von Humboldt foundation for financial support. 


\section{A Matrix models with $\mathbf{S O}(1,2)$ and gauge symmetries}

Most of the matrix models we consider have $\mathrm{SO}(1,2)$ symmetry. Here we discuss how the symmetry is realized.

To begin, we review the so(1,2) structure behind a second order differential equation [25]. For a given arbitrary time dependent function, $\Lambda(t)$, we consider the following second order differential equation,

$$
\ddot{f}(t)=f(t) \Lambda(t) .
$$

Naturally there are two distinct solutions and we denote them by $f_{+}(t)$ and $f_{-}(t)$. Since

$$
\frac{\mathrm{d}}{\mathrm{d} t}\left(f_{+} \dot{f}_{-}-f_{-} \dot{f}_{+}\right)=0
$$

if we set a non-zero constant,

$$
c:=f_{+}(t) \dot{f}_{-}(t)-f_{-}(t) \dot{f}_{+}(t) \neq 0,
$$

and define

$$
J_{0}:=-i \frac{1}{2 c}\left(f_{+}^{2}+f_{-}^{2}\right) \partial_{t}, \quad J_{1}:=-i \frac{1}{2 c}\left(f_{+}^{2}-f_{-}^{2}\right) \partial_{t}, \quad J_{2}:=-i \frac{1}{c} f_{+} f_{-} \partial_{t},
$$

then the $\mathbf{s p}(2, \mathbf{R}) \equiv \mathrm{so}(1,2) \equiv \mathbf{s l}(2, \mathbf{R})$ Lie algebra follows in the standard form

$$
\left[J_{0}, J_{1}\right]=+i J_{2}, \quad\left[J_{1}, J_{2}\right]=-i J_{0}, \quad\left[J_{2}, J_{0}\right]=+i J_{1} .
$$

It is useful to note that the induced infinitesimal transformations, $\delta t$, generated by $i J_{k}$ can be equivalently specified as the three general solutions of the following third order differential equation,

$$
\frac{\mathrm{d}^{3} \delta t}{\mathrm{~d} t^{3}}=4 \Lambda \frac{\mathrm{d} \delta t}{\mathrm{~d} t}+2 \frac{\mathrm{d} \Lambda}{\mathrm{d} t} \delta t .
$$

Further, we introduce one more arbitrary time dependent function $\rho(t)$, and consider the following second order differential equation,

$$
\ddot{\chi}(t)=\Lambda(t) \chi(t)+\rho(t) .
$$

In order to write down the general solution explicitly in terms of $f_{ \pm}(t)$, it is convenient to define, with arbitrary constants, $\kappa_{ \pm}(0)$,

$$
\kappa_{+}(t):=\kappa_{+}(0)+\int_{0}^{t} \mathrm{~d} t^{\prime} \rho\left(t^{\prime}\right) f_{+}\left(t^{\prime}\right), \quad \kappa_{-}(t):=\kappa_{-}(0)+\int_{0}^{t} \mathrm{~d} t^{\prime} \rho\left(t^{\prime}\right) f_{-}\left(t^{\prime}\right) .
$$


Then the most general solution reads explicitly,

$$
\chi(t)=\frac{1}{c}\left(f_{-}(t) \kappa_{+}(t)-f_{+}(t) \kappa_{-}(t)\right) .
$$

The arbitrary constants, $\kappa_{ \pm}(0)$, amount to the homogeneous solutions to (A.7).

Now we consider the following $N \times N$ matrix model with $\mathrm{U}(N)$ gauge symmetry,

$$
\mathcal{L}_{\mathrm{SO}(1,2)}=\operatorname{tr}\left[\frac{1}{2}\left(D_{t} X\right)^{2}+\frac{1}{2} \Lambda(t) X^{2}+\rho(t) X+\frac{2 v}{(X-\chi(t) 1)^{2}}\right],
$$

where $v$ is a constant, and $\Lambda(t), \rho(t)$ are arbitrary time dependent coefficients, while $\chi(t)$ is $a$ solution of the second order differential equation (A.7).

The crucial fact is that there exits $\mathrm{SO}(1,2)$ symmetry in the matrix model generated by the following infinitesimal transformation, ${ }^{10}$

$$
\delta X=\delta t D_{t} X-\frac{1}{2} \dot{\delta} t X-\zeta(t) 1, \quad \delta A=0 .
$$

The diffeomorphism, $\delta t$, is generated by $i J_{k}$ A.4 above, or equivalently as general solutions of the third order differential equation (A.6). The inhomogeneous term is given for each $\delta t$ by

$$
\zeta(t)=\delta t \dot{\chi}(t)-\frac{1}{2} \dot{\delta} t \chi(t) .
$$

$X$ is a quasi-primary operator of the "conformal" weight $1 / 2$.

Especially, in the absence of the inverse square potential, i.e. $v=0$, we do not need to fix $\chi(t)$ as a solution of the second order differential equation (A.7). Consequently there are two parameter freedom in the inhomogeneous term, which amounts to the following extra symmetries, ${ }^{11}$

$$
\delta X=f_{+}(t) 1 \quad \text { and } \quad \delta X=f_{-}(t) 1 .
$$

Turning on the inverse square potential, $v \neq 0$, these extra symmetries are all broken.

It is worth to note that when $\Lambda$ and $\rho$ are constants, we have $\chi(t)=-\rho \Lambda^{-1}+a f_{+}(t)+b f_{-}(t)$ with two arbitrary constants $a, b$. Only if these vanish, the system corresponds to the constant

\footnotetext{
${ }^{10}$ In general, $\delta A$ can be an arbitrary linear combination of $D_{t} X$ and $X$, and here for simplicity we put $\delta A \equiv 0$. However, in the supersymmetric extension of the above matrix model it is required to set $\delta X=\delta A$ and $v=0$ [25].

${ }^{11}$ Hence, when $v=0$, the inhomogeneous term can be alternatively given as the general solutions of

$$
\ddot{\zeta}=\Lambda \zeta+\frac{3}{2} \rho \dot{\delta} t+\dot{\rho} \delta t
$$

and the extra symmetries A.13 correspond to the homogeneous part of the solutions [25].
} 
shift of $X$ by $\rho \Lambda^{-1}$ from the $\rho=\chi=0$ system. If $\Lambda$ is a positive constant and $\rho=\chi=0$, it corresponds to the 'deformed matrix model' [26]; if $\Lambda$ is a positive constant and $\rho=v=0$, it amounts to the so(1,2) subalgebra of the $W_{\infty}$ algebra [36]; if $\Lambda=0$, then $\delta t$ is quadratic in $t$, and the $\mathrm{SO}(1,2)$ symmetry can be identified as a conformal symmetry. With one more condition $\rho=0$, it reduces to the well known conformal matrix model [51].

Introducing an auxiliary matrix, $F$, we can rewrite the Lagrangian (A.14) in a non-singular manner,

$$
\mathcal{L}_{\mathrm{SO}(1,2)}^{\prime}=\operatorname{tr}\left[\frac{1}{2}\left(D_{t} X\right)^{2}+\frac{1}{2}\left(\Lambda(t)-F^{2}\right) X^{2}+\left(\rho(t)+\chi(t) F^{2}\right) X-\frac{1}{2} \chi(t)^{2} F^{2}+2 \sqrt{v} F\right] .
$$

However, our analysis in Sec. 3.2 shows that the inverse square potential is absent in the matrix model formulation of the DBI action in any isometric background. 


\section{References}

[1] J. M. Maldacena, Adv. Theor. Math. Phys. 2, 231 (1998) [Int. J. Theor. Phys. 38, 1113 (1999)] arXiv:hep-th/9711200.

[2] O. Aharony, S. S. Gubser, J. M. Maldacena, H. Ooguri and Y. Oz, Phys. Rept. 323, 183 (2000) arXiv:hep-th/9905111.

[3] M. E. Olsson, JHEP 0605 (2006) 032 arXiv:hep-th/0511106.

[4] M. R. Douglas, I. R. Klebanov, D. Kutasov, J. Maldacena, E. Martinec and N. Seiberg, "A new hat for the $\mathrm{c}=1$ matrix model," arXiv:hep-th/0307195.

[5] I. R. Klebanov, J. Maldacena and N. Seiberg, JHEP 0307, 045 (2003) arXiv:hep-th/0305159.

[6] J. M. Lapan and W. Li, "Falling D0-branes in 2D superstring theory," arXiv:hep-th/0501054.

[7] D. Kutasov, "Accelerating branes and the string / black hole transition," arXiv:hep-th/0509170

[8] L. Susskind, Phys. Rev. D 49 (1994) 6606 arXiv:hep-th/9308139.

[9] L. Susskind, arXiv:hep-th/9309145.

[10] L. Susskind and J. Lindesay, "An introduction to black holes, information and the string theory revolution: The holographic universe," World Scientific Publishing Co. (2005).

[11] G. T. Horowitz and J. Polchinski, Phys. Rev. D 55 (1997) 6189 arXiv:hep-th/9612146.

[12] A. Giveon, D. Kutasov, E. Rabinovici and A. Sever, Nucl. Phys. B 719 (2005) 3 arXiv:hep-th/0503121.

[13] Y. Nakayama, S. J. Rey and Y. Sugawara, JHEP 0509 (2005) 020 hep-th/0507040.

[14] A. Giveon and D. Kutasov, JHEP 0601 (2006) 120 arXiv:hep-th/0510211.

[15] A. Parnachev and D. A. Sahakyan, arXiv:hep-th/0512075.

[16] J. M. Maldacena and A. Strominger, JHEP 9712 (1997) 008 arXiv:hep-th/9710014.

[17] V. Kazakov, I. K. Kostov and D. Kutasov, Nucl. Phys. B 622 (2002) 141 arXiv:hep-th/0101011.

[18] D. Kutasov, "D-brane dynamics near NS5-branes," arXiv:hep-th/0405058.

[19] J. L. Karczmarek, J. Maldacena and A. Strominger, JHEP 0601 (2006) 039 arXiv:hep-th/0411174. 
[20] D. M. Thompson, Phys. Rev. D 70 (2004) 106001 arXiv:hep-th/0312156.

[21] T. Suyama and P. Yi, JHEP 0402 (2004) 017 arXiv:hep-th/0401078.

[22] J. L. Davis, L. A. Pando Zayas and D. Vaman, JHEP 0403 (2004) 007 arXiv:hep-th/0402152.

[23] U. H. Danielsson, J. P. Gregory, M. E. Olsson, P. Rajan and M. Vonk, JHEP 0404 (2004) 065 arXiv:hep-th/0402192.

[24] J. L. Davis and R. McNees, JHEP 0509 (2005) 072 arXiv:hep-th/0411121.

[25] J.-H. Park, Nucl. Phys. B 745 (2006) 123 arXiv:hep-th/0510070.

[26] A. Jevicki and T. Yoneya, Nucl. Phys. B 411 (1994) 64 arXiv:hep-th/9305109.

[27] A. Strominger, JHEP 0403 (2004) 066 arXiv:hep-th/0312194.

[28] S. Gukov, T. Takayanagi and N. Toumbas, JHEP 0403 (2004) 017 arXiv:hep-th/0312208.

[29] T. Banks and M. O'Loughlin, Phys. Rev. D 48, 698 (1993) arXiv:hep-th/9212136.

[30] N. Berkovits, S. Gukov and B. C. Vallilo, Nucl. Phys. B 614, 195 (2001) arXiv:hep-th/0107140.

[31] S. N. Solodukhin, Phys. Rev. D 53 (1996) 824 arXiv:hep-th/9506206.

[32] G. Semenoff, private communication.

[33] E. Witten, Nucl. Phys. B 460 (1996) 335 arXiv:hep-th/9510135.

[34] J.-H. Park, Phys. Lett. A 307 (2003) 183 arXiv:hep-th/0203017.

[35] J. McGreevy and H. L. Verlinde, JHEP 0312, 054 (2003) arXiv:hep-th/0304224.

[36] J. Avan and A. Jevicki, Phys. Lett. B 266, 35 (1991);

J. Avan and A. Jevicki, Phys. Lett. B 272, 17 (1991);

S. R. Das, A. Dhar, G. Mandal and S. R. Wadia, Int. J. Mod. Phys. A 7, 5165 (1992);

S. R. Das, A. Dhar, G. Mandal and S. R. Wadia, Mod. Phys. Lett. A 7, 71 (1992);

E. Witten, Nucl. Phys. B 373, 187 (1992).

[37] T. Takayanagi, private communication.

[38] I. R. Klebanov, "String theory in two-dimensions," arXiv:hep-th/9108019.

[39] P. H. Ginsparg and G. W. Moore, "Lectures on 2-D gravity and 2-D string theory," arXiv:hep-th/9304011 
[40] J. Polchinski, "What is string theory?," arXiv:hep-th/9411028.

[41] J. McGreevy, J. Teschner and H. L. Verlinde, JHEP 0401 (2004) 039 arXiv:hep-th/0305194.

[42] T. Takayanagi and N. Toumbas, JHEP 0307, 064 (2003) arXiv:hep-th/0307083.

[43] T. Takayanagi, JHEP 0411 (2004) 030 arXiv:hep-th/0408086.

[44] T. Takayanagi and S. Terashima, JHEP 0506 (2005) 074 arXiv:hep-th/0503184.

[45] J. Maldacena and N. Seiberg, JHEP 0509 (2005) 077 arXiv:hep-th/0506141.

[46] J. McGreevy, S. Murthy and H. L. Verlinde, JHEP 0404 (2004) 015 arXiv:hep-th/0308105.

[47] P. M. Ho, JHEP 0405, 008 (2004) arXiv:hep-th/0401167.

[48] O. Aharony and A. Patir, JHEP 0511 (2005) 052 arXiv:hep-th/0509221.

[49] M. Spradlin and A. Strominger, JHEP 9911, 021 (1999) arXiv:hep-th/9904143.

[50] L. P. Kadanoff, "Statistical Physics: Statics, Dynamics And Renormalization," World Scientific (2000).

[51] V. de Alfaro, S. Fubini and G. Furlan, Nuovo Cim. A 34, 569 (1976). 